This manuscript is a post print and has been accepted for publication in Physics of the Earth and Planetary Interiors special volume: Physical properties and observations of the lithosphere-asthenosphere system. Subsequent versions of this manuscript may be slightly different. The final version of this manuscript will be available: Publication DOI: https://doi.org/10.1016/j.pepi.2020.106486. Please feel free to contact any of the authors; we welcome feedback. 


\title{
The influence of spreading rate and permeability on melt focusing beneath mid-ocean ridges
}

\author{
Shi J. Sim ${ }^{\mathrm{a}, \mathrm{d}}$, Marc Spiegelman ${ }^{\mathrm{b}, \mathrm{c}}$, Dave R. Stegman ${ }^{\mathrm{a}}$, Cian Wilson ${ }^{\mathrm{d}}$ \\ ${ }^{a}$ Institute for Geophysics and Planetary Physics, Scripps Institution of Oceanography, \\ University of California San Diego, La Jolla, California, USA \\ ${ }^{b}$ Department of Earth and Environmental Sciences, Columbia University, New York, \\ New York, USA \\ ${ }^{c}$ Applied Physics and Applied Mathematics, Columbia University, New York, New York, \\ USA \\ ${ }^{d}$ Department of Terrestrial Magnetism, Carnegie Institution for Science, Washington, \\ District of Columbia, USA
}

\section{Abstract}

At mid-ocean ridges, oceanic crust is emplaced in a narrow neo-volcanic region on the seafloor, whereas basaltic melt that forms this oceanic crust is generated in a wide region beneath as suggested by a few geophysical surveys. The combined observations suggest that melt generated in a wide region at depths has to be transported horizontally to a small region at the surface. We present results from a suite of two-phase models applied to the mid-ocean ridges, varying half-spreading rate and intrinsic mantle permeability using new openly available models, with the goal of understanding melt focusing beneath mid-ocean ridges and its relevance to the lithosphere-asthenosphere boundary (LAB). Three distinct melt focusing mechanisms are recognized in these models: 1) melting pressure focusing, 2) decompaction layers and 3) ridge suction, of which the first two play dominant roles in focusing melt. All

Email address: ssim@carnegiescience.edu () 
three of these mechanisms exist in the fundamental two phase flow formulation but the manifestation depends largely on the choice of rheological model. The models also show that regardless of spreading rates, the amount of melt and melt transport patterns are sensitive to changes in intrinsic permeability, $K_{0}$. In these models, the LAB is delineated by the melt-rich decompaction layers, which are essentially defined by the temperature dependent rheological and freezing boundaries. Geophysical observations place the LAB at a steeper incline as compared to the gentler profile suggested by most of our models. The models suggest that one way to reconcile this discrepancy is to have stronger melting pressure focusing mechanism as it is the only mechanism in these models that can focus melt before reaching the typical model thermal LAB. The apparent lack of observable decompaction layers in the geophysical observations hints at the possibility that melting pressure focusing could be significant. These models help improve our understanding of melt focusing beneath mid-ocean ridges and could provide new constraints for mantle rheology and permeability.

Keywords: two-phase flow, mid-ocean ridges, geodynamics, melt transport in the mantle, melt focusing, lithosphere asthenosphere boundary

\section{Introduction}

2 Mid-ocean ridges are a prominent feature of plate tectonics that run for 3 more than $60,000 \mathrm{~km}$ on the ocean floor. Tectonic forces pull oceanic plates 4 apart at ridges, allowing mantle to upwell and generate melt due to decom5 pression melting. New oceanic crust is created at a narrow neo-volcanic 6 region a few kilometers across, accounting for $60 \%$ of global magmatism 
7 (Macdonald, 1982; Vera et al., 1990; Carbotte et al., 2016). Geophysical s observations at specific mid-ocean ridges suggest that there are regions of 9 partially molten mantle a few hundred kilometers wide at $\sim 50 \mathrm{~km}$ depths 10 (Forsyth et al., 1998b,a; Key et al., 2013). Without any external forcing,

buoyant melt will rise vertically due to the density contrast with the background mantle. The combined observations of a wide region of melt generation and narrow volcanic zone suggest that mechanisms have to be present to move melt horizontally (or simply melt focusing).

Several processes have been proposed to explain melt focusing, of which ridge suction and decompaction layers are well established. 'Ridge suction' is the melt flow driven by a reduction in dynamic pressure due to incompressible shear near the ridge axis which pulls melt towards the ridge axis from the surrounding region (Spiegelman and McKenzie, 1987; Morgan, 1987). This pressure gradient becomes strong when the solid mantle is assumed to be highly viscous. For melt focusing by decompaction layers (Sparks and Parmentier, 1991; Spiegelman, 1993c; Hebert and Montési, 2010; Keller et al., 2017), buoyant melt is generated in a wide melting region and travels upwards until it encounters an impermeable barrier formed by the thermal boundary layer of the cold and stronger lithosphere. Compaction pressure opens up pore space beneath this impermeable barrier, where melt can accumulate and pond. The melt in these decompaction layers or high porosity "channels" then travels along the slope of the lithosphere towards the ridge axis. Melting pressure focusing is a recently recognized mechanism (Turner et al., 2017), in which compaction pressure focuses melt if bulk viscosity and melting rates are sufficiently large in quasi-steady state. This study focuses on 
these three melt focusing mechanisms. Other focusing mechanisms have also been proposed that we do not consider. Anisotropic permeability generated by dike propagation (Morgan, 1987) could focus melt, although the mode of dike propagation proposed would emplace melt away from the ridge axis. A related mechanism to dike propagation is the formation of shear driven melt bands from localization instabilities that align melt bands in accordance to the shear direction (Holtzman et al., 2003b,a; Katz et al., 2006; Kohlstedt and Holtzman, 2009).

In this study, we not only highlight and explain the three melt focusing mechanisms as laid out above, but also demonstrate the robustness of the melting pressure mechanism without any complexity of grain size evolution as included in Turner et al. (2017). Studies of melting and melt transport at mid-ocean ridges have used one-dimensional column modeling (Ribe, 1985; Asimow and Stolper, 1999; Hewitt and Fowler, 2008) whereas twodimensional modeling efforts (including work presented here) show that horizontal melt transport is inherent to the magma migration formulation in the mid-ocean ridge setting (Buck and Su, 1989; Spiegelman, 1993c; Hebert and Montési, 2010; Keller et al., 2017). Recently, other two dimensional models use similar two phase formulation employing reactive transport (Keller and Katz, 2016; Keller et al., 2017) to understand volatile distribution and incorporating grain size evolution to explore melt focusing (Turner et al., 2017). Both melting pressure focusing and decompaction layers will not be present in models that exclude pressure gradients due to viscous solid deformation (e.g. Buck and Su (1989); Scott and Stevenson (1984)).

To investigate melt focusing mechanisms and its relation to the lithosphere- 
asthenosphere boundary (LAB), we present a suite of new open-source twodimensional two-phase flow models and explore the primary controls of spreading rate, $U_{0}$, and intrinsic permeability, $K_{0}$. These models are built using TerraFERMA, the Transparent Finite Element Rapid Model Assembler (Wilson et al., 2017), and solves the melt migration equations that were derived independently by several workers (McKenzie, 1984; Fowler, 1985; Scott and Stevenson, 1986). The spreading rate is a fundamental observation at midocean ridges. The permeability of the solid mantle depends on grain size and is coupled with the porosity, melt transport and length scale at which the mantle promotes/resists melt transport. Larger permeability promotes faster segregation of melt and therefore smaller melt retention, and vice versa. The retention of melt affects the bulk viscosity, which alters the strength of the melting pressure focusing mechanism, such that melting pressure focusing will be weaker with more melt retained. Our new models include thermal evolution, melting that evolves depending on pressure, temperature and nonlinear solid rheology that depends on temperature and strain-rate as compared to recent modeling efforts with reactive transport, Newtonian rheology and constant grain size (Keller et al., 2017) or with grain size evolution and non-Newtonian rheology (Turner et al., 2017). We also explore how different rheological choices for shear viscosity affects both decompaction layers and melting pressure focusing effect. Both TerraFERMA and the model description files are open source and available at terraferma.github.io.

The LAB can be defined multiple ways, e.g. as a thermal, rheological, permeability and melt-rich boundary etc (Fischer et al., 2010). The LAB is not always clearly defined by geophysical observations. Seismic velocities 
are sensitive to several physical properties such as temperature, melt, grain size and density etc (Kawakatsu and Utada, 2017). Similarly, electromagnetic methods are sensitive to temperature, hydration, melt, oxygen fugacity and chemical composition (Kawakatsu and Utada, 2017). Although geophysical inversions are often non-unique, even when the velocity or conductivity structures are known exactly, the interpretation can lead to tradeoffs between the physical properties. Nevertheless, geophysical observations provide good constraints when combined with independent information. This is where modeling can come in handy to understand the underlying processes and properties from a different perspective. If the LAB is delineated by a melt-no melt boundary, then there are some disagreements between observations and models about the location of the LAB at mid-ocean ridges. In two-phase flow models, both rheological strengthening with temperaturedependent rheology and crystallization can lead to a melt-rich decompaction layer that follows the predicted shallow thermal LAB. Both the steep-sided conductive partial melt region shown by electromagnetic survey at the East Pacific Rise (Key et al., 2013), and the deeper than expected large seismic attenuating region within $50 \mathrm{~km}$ of the ridge axis beneath the Juan de Fuca Ridge (Eilon and Abers, 2017; Ruan et al., 2018) suggest that melt focusing has to occur before melt can reach the shallower thermal LAB or the meltrich decompaction layers in our models. Melting pressure focusing is the only mechanism so far that can focus melt before melt reaches the shallow LAB.

Section 2 describes the model set up, laying out the one-way coupled non-dimensional form of the magma migration equations along with closure equations (rheology, interphase exchange and permeability), the boundary 
and initial conditions chosen, with all the parameters and variables given in Tables 1 and 2. Section 3 describes the general evolution of the models and first order trends such as porosity or melt fraction and melt transport with respect to our variables, half spreading rates and intrinsic permeability. We predict crustal thickness using proxies from the model outputs and show how they fit with crustal thickness from geophysical observations. The discussion section goes through the three recognized melt focusing mechanisms in terms of the associated pressures. We also present models with different rheological model choices for shear viscosity to illustrate melting pressure focusing mechanism. Then, we analyze the melt focusing trends versus spreading rate and permeability using proportion of melt flux focused by the dominant mechanisms. We compare the location and amount of melt in the model results with geophysical observations and try to reconcile the difference in the understanding of the LAB between the two. Finally, we discuss the limitations of our model setup and conclude the findings.

\section{Model formulation}

We model melt migration beneath a mid-ocean ridge using a Darcy-like melt flow in a viscously deforming solid matrix (McKenzie, 1984; Scott and Stevenson, 1984, 1986; Fowler, 1985). To clearly identify the various pressure gradients that affect melt flow, we decompose the total liquid pressure into compaction pressure, $\mathcal{P}$, dynamic pressure, $p^{*}$, and lithostatic pressure, $P_{L}$ (Spiegelman, 1993c; Katz et al., 2007; Keller and Katz, 2016):

$$
P=\mathcal{P}+p^{*}+P_{L}
$$


Compaction pressure, $\mathcal{P}$, is defined by the divergence of solid velocity, $\mathbf{v}_{\mathbf{s}}$, or the compaction and decompaction of the solid matrix scaled by the bulk viscosity and is a measure of the pressure difference between the phases:

$$
\mathcal{P}=\zeta \nabla \cdot \mathbf{v}_{\mathbf{s}}
$$

This definition serves to indicate an overpressure when the divergence of solid velocity is positive, which is consistent with the idea of melt overpressure driving expansion of the matrix, and underpressure causing compaction (McKenzie, 1984). The definition of compaction pressure here follows previous work such as Katz (2008) while it differs in sign from Keller et al. (2017). $p^{*}$ is the dynamic pressure due to incompressible solid shear and $P_{L}$ is a reference lithostatic pressure:

$$
P_{L}(z)=\rho_{s} g z
$$

Substituting the pressure decomposition into the dimensional Darcy's equation, we have:

$$
\phi\left(\mathbf{v}_{\mathbf{f}}-\mathbf{v}_{\mathbf{s}}\right)=-\frac{K}{\mu}\left[\nabla \mathcal{P}+\nabla p^{*}+\Delta \rho \mathbf{g}\right]
$$

where $\phi$ is the porosity, the volume fraction of the melt of liquid phase in the two-phase system. We assume that any pore space in the solid matrix is saturated with melt. $\mathbf{v}_{\mathbf{s}}$ and $\mathbf{v}_{\mathbf{f}}$ are the solid and liquid velocities, $K$ is the matrix permeability and $\mu$ is the liquid viscosity. This equation says that melt segregation occurs due to various pressure gradients modulated by permeability and liquid viscosity. The buoyancy term moves melt vertically. Since we are exploring melt focusing in this study, the horizontal pressure gradients would be of interest although the pressures can also transport melt vertically. 
Compaction and dynamic pressures focus melt if their corresponding gradients in the horizontal direction is significant compared to buoyancy. In this work, the set of equations is non-dimensionalised with velocities scaled to the reference melt separation velocity, lengths scaled to the depth of the domain, pressures scaled to the lithostatic pressure of the depth of the domain and temperature scaled to the mantle potential temperature. The full derivation and non-dimensionalization are given in the supplementary material. The characteristic scales used are give in Table 1.

In the limit of small porosity, Spiegelman $(1993 \mathrm{a}, \mathrm{b})$ show that the momentum and continuity equations of the solid and liquid become decoupled such that the solid flow no longer depends on the liquid flow, therefore allowing us to use the one-way coupling approach, e.g. Wilson et al. (2014). First, we solve the time-independent solid Stokes flow and the steady-state energy equation (Equations 5-7) once at the beginning of each model run for solid velocity and dynamic pressure and temperature. Holding the solid flow constant (i.e. the solid velocity and dynamic pressure solution does not change with time) and using the temperature solution from the solid solve as the initial condition, we then solve for the time-dependent two-phase flow (Equations 15-17) for porosity, compaction pressure and temperature. Both solid and two-phase systems are solved in a two-dimensional rectangular domain $200 \mathrm{~km}$ wide and $100 \mathrm{~km}$ deep as shown in Figure 1, where the center line $(\mathrm{x}=0)$ represents the ridge axis and the top of the domain $(\mathrm{z}=0)$ represents the base of the Moho. 


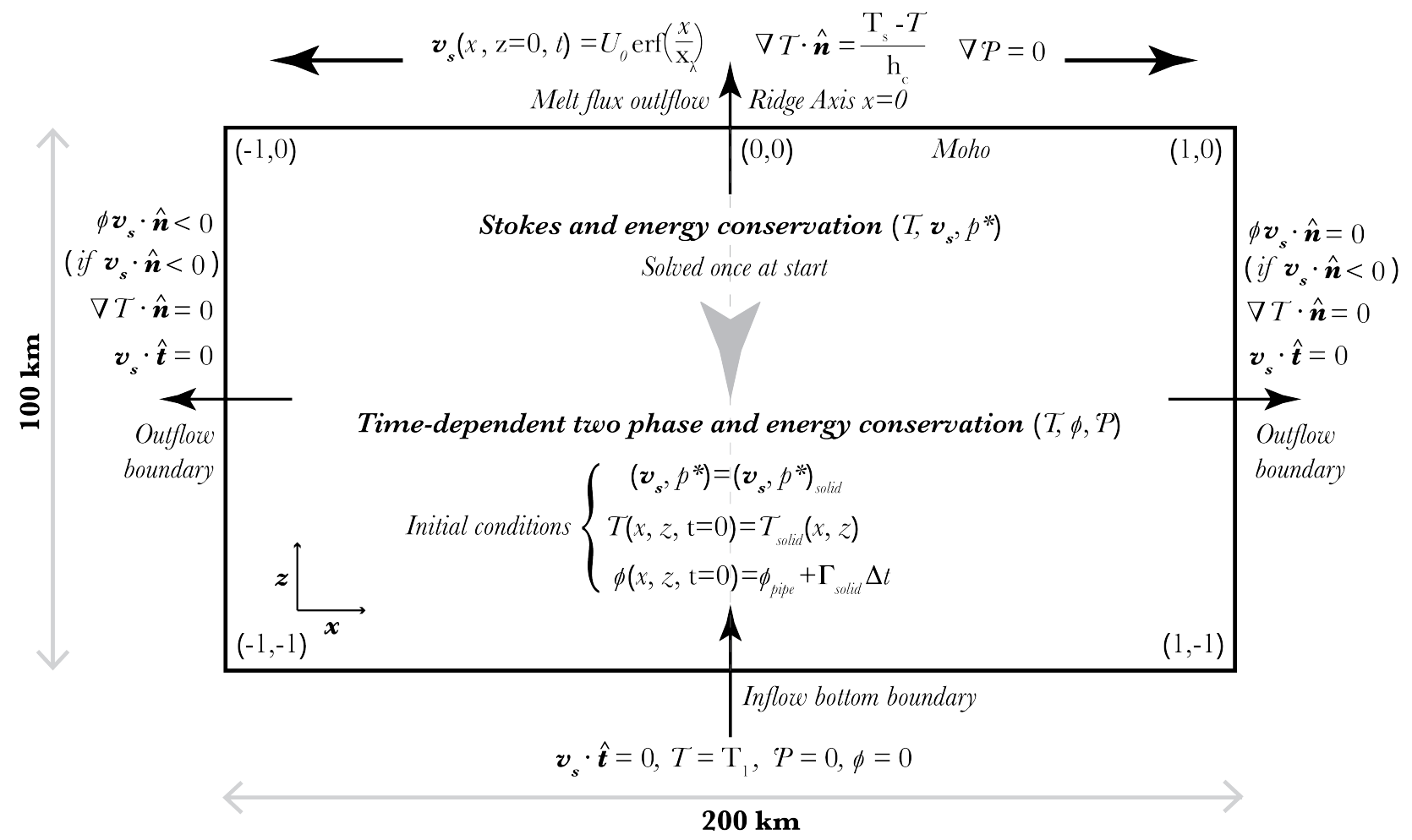

Figure 1: Schematic of two-dimensional model diagram with initial and boundary conditions. 
175

176

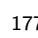

184

185

\subsection{Solid Stokes system}

In the solid flow system, the non-dimensionalized incompressible Stokes equations are solved along with the steady-state thermal energy equation:

$$
\phi_{0}^{m-1} \frac{\delta_{0}^{2}}{h^{2}} \nabla \cdot 2 \eta \dot{\epsilon}_{d}-\nabla p^{*}=0
$$

$$
\nabla \cdot \mathbf{v}_{\mathbf{s}}=0
$$

$$
\mathbf{v}_{\mathbf{s}} \cdot \nabla T-\beta T \mathbf{v}_{\mathbf{s}} \cdot \hat{\mathbf{k}}+\phi_{0} L_{c p} T \Gamma_{\text {Stokes }}-\frac{1}{P e} \nabla^{2} T=0
$$

where we solve for the dynamic pressure, $p^{*}$, the velocity of the solid phase, $\mathbf{v}_{\mathbf{s}}$, and the temperature, T. $m$ is the bulk viscosity exponent, $h$ is the depth of the domain, $\beta$ is the non-dimensional adiabatic gradient, $L_{c p}$ is the nondimensional latent heat coefficient and $P e$ is the Peclet number. We define the reference porosity, $\phi_{0}$, and melt velocity, $w_{0}$, using a buoyancy-driven Darcy-flow approximation and mass conservation for a one-dimensional melt column (Spiegelman and Elliott, 1993; Ribe, 1987) given by:

$$
\phi_{0} w_{0}=\frac{K_{0} \phi_{0}^{n} \Delta \rho g}{\mu_{0}}
$$

and

$$
\rho_{f} \phi_{0} w_{0}=\rho_{s} U_{0} F_{\max }
$$

${ }_{185}$ The reference compaction length, $\delta_{0}$, is defined as:

$$
\delta_{0}=\sqrt{\frac{K_{0} \phi_{0}^{n} \eta_{0}}{\mu_{0} \phi_{0}^{m}}}
$$

${ }_{186} \dot{\epsilon}_{d}=\frac{1}{2}\left(\nabla \mathbf{v}_{\mathbf{s}}+\nabla \mathbf{v}_{\mathbf{s}}^{\mathbf{T}}\right)-\frac{1}{3} \nabla \cdot \mathbf{v}_{\mathbf{s}} \mathbf{I}$ is the deviatoric strain rate tensor. $\eta$ is the non-dimensional solid shear viscosity, given by a harmonic sum of diffusion 
creep, dislocation creep and a small plasticity term to keep the ridge axis weak (Spiegelman et al., 2016; Tosi et al., 2015):

$$
\frac{1}{\eta}=\frac{\eta_{0}}{\eta_{\text {diff }}(T)}+\frac{\eta_{0}}{\eta_{\text {disl }}\left(T, \dot{\epsilon}_{\mathrm{II}}\right)}+\frac{\eta_{0}}{\eta_{\text {plas }}\left(\dot{\epsilon}_{\mathrm{II}}\right)}+\frac{\eta_{0}}{\eta_{\max }}
$$

where diffusion creep, dislocation creep (Karato and Wu, 1993; Hirth and Kohlstedt, 2003) and the plasticity term are given by:

$$
\eta_{\text {diff }}=A_{\text {diff }} e^{\left[E_{\text {diff }} /(n R T)\right]}
$$

$$
\eta_{\text {disl }}=A_{\text {disl }} e^{\left[E_{\text {disl }} /(n R T)\right]}{\frac{1}{\hat{\epsilon}_{I I}}}^{\frac{1}{n_{\text {disl }}}-1}
$$

and

$$
\eta_{\text {plas }}=\frac{Y}{2 \dot{\epsilon}_{\mathrm{II}}}
$$

$A_{\text {diff }}$ and $E_{\text {diff }}$ are the constant and activation energy for diffusion creep respectively, and $R$ is the universal gas constant. $A_{\text {disl }}$ and $E_{\text {disl }}$ are the constant and activation energy for dislocation creep respectively, $\dot{\epsilon}_{\text {II }}$ is the second invariant of strain rate and $n_{\text {disl }}$ is the stress exponent. $Y=C \cos \varphi+P_{L} \sin \varphi$ is the yield criterion, $C$ is the constant of cohesion independent of pressure, and $\varphi$ is the friction angle. $\Gamma_{\text {Stokes }}$ is the non-dimensional interphase mass exchange rate. Despite not including the melt phase in the solid Stokes equation, the melting term in the energy equation is not dropped in the small porosity approximation since it is of order $F_{\max }$, the maximum degree of melting, when taking into account that $\Gamma_{\text {Stokes }}$ is of order $F_{\max } / \phi_{0}$.

\subsection{Two-phase system}

The mantle upwelling from the solid system drives decompression melting. We model the evolution of this melt using the non-dimensionalized two-phase 
Table 1: Symbols and definitions of parameters

\begin{tabular}{|c|c|c|c|}
\hline Symbol & Formula & Definition & Value \\
\hline$h$ & & reference length scale & $100 \mathrm{~km}$ \\
\hline$T_{0}$ & & reference mantle temperature & $1623 \mathrm{~K}$ \\
\hline$\rho_{s}$ & & density of solid phase & $3300 \mathrm{~kg} / \mathrm{m}^{3}$ \\
\hline$\rho_{f}$ & & density of liquid phase & $2800 \mathrm{~kg} / \mathrm{m}^{3}$ \\
\hline$\Delta \rho$ & $\rho_{s}-\rho_{f}$ & density difference between solid and liquid phases & $500 \mathrm{~kg} / \mathrm{m}^{3}$ \\
\hline$F_{\max }$ & & maximum degree of melting & 0.2 \\
\hline$\mu_{0}$ & & reference liquid viscosity & $1 \mathrm{~Pa} \mathrm{~s}$ \\
\hline$\eta_{0}$ & & reference background solid shear viscosity & $10^{19} \mathrm{~Pa} \mathrm{~s}$ \\
\hline$\eta_{\max }$ & & maximum solid shear viscosity & $10^{23} \mathrm{~Pa} \mathrm{~s}$ \\
\hline$\beta$ & $\alpha_{s} g h / c_{p}$ & non-dimensional adiabatic gradient & $2.45 \times 10^{8}$ \\
\hline$\alpha_{s}$ & & thermal expansion coefficient for solid phase & $3 \times 10^{5} / \mathrm{K}$ \\
\hline$g$ & & gravitational acceleration & $9.81 \mathrm{~m} / \mathrm{s}^{2}$ \\
\hline$c_{p}$ & & heat capacity at constant pressure for solid phase & $1200 \mathrm{~J} / \mathrm{K}$ \\
\hline$L_{c p}$ & $L_{0} / T_{0} c_{p}$ & non-dimensional latent heat & 0.205 \\
\hline$L_{0}$ & & reference latent heat of melting & $4 \times 10^{5} \mathrm{~J} / \mathrm{kg}$ \\
\hline$\kappa_{0}$ & $k / \rho_{s} c_{p}$ & reference thermal diffusivity & $7.272 \times 10^{-7} \mathrm{~m}^{2} / \mathrm{s}$ \\
\hline$A_{\text {diff }}$ & & Diffusion creep constant & $1.32043 \times 10^{9}$ \\
\hline$E_{\text {diff }}$ & & Activation energy for diffusion creep & $335 \times 10^{3} \mathrm{~J}$ \\
\hline$A_{\text {disl }}$ & & Dislocation creep constant & 28968.6 \\
\hline$E_{\text {disl }}$ & & Activation energy for dislocation creep & $540 \times 10^{3} \mathrm{~J}$ \\
\hline$R$ & & Universal gas constant & $8.314 \mathrm{~J} / \mathrm{K} \mathrm{mol}$ \\
\hline$n_{\text {disl }}$ & & exponent dependence on strain rate for dislocation creep & 3.5 \\
\hline$C$ & & constant of cohesion independent of pressure & $100 \mathrm{MPa}$ \\
\hline$\varphi$ & & friction angle for yield criterion & $30^{\circ}$ \\
\hline$m$ & & bulk viscosity exponent & 1 \\
\hline$\phi_{\epsilon}$ & & regularization for porosity in bulk viscosity relation & 0.01 \\
\hline$A_{1}, A_{2}, A_{3}$ & & constants for peridotite solidus & $1085.7^{\circ} \mathrm{C}, 132.9^{\circ} \mathrm{C} / \mathrm{GPa},-5.1^{\circ} \mathrm{C} / \mathrm{GPa}^{2}$ \\
\hline$B_{1}, B_{2}, B_{3}$ & & constants for peridotite liquidus & $1475.0{ }^{\circ} \mathrm{C}, 80.0{ }^{\circ} \mathrm{C} / \mathrm{GPa},-3.2{ }^{\circ} \mathrm{C} / \mathrm{GPa}^{2}$ \\
\hline$n$ & & permeability exponent & 3 \\
\hline$\Delta t$ & & Time step for initial porosity estimation & 0.1 \\
\hline$\theta_{\mathrm{tr}}$ & & degree of fanning downwards for width of initial porosity transient & 100 \\
\hline$x_{\text {width }}$ & & non-dimensional width of initial porosity transient at ridge axis & 0.01 \\
\hline$z_{0}$ & & non-dimensional beginning depth of initial porosity transient & 0 \\
\hline$z_{1}$ & & non-dimensional ending depth of initial porosity transient & 0.1 \\
\hline$z_{\lambda}$ & & non-dimensional width of vertical smoothing for initial porosity transient & 0.035 \\
\hline
\end{tabular}


Table 2: Symbols for variables and their definition

\begin{tabular}{|c|c|c|c|c|c|c|c|c|c|}
\hline Symbol & Formula & Definition & \multicolumn{6}{|c|}{ Values } & Units \\
\hline$K_{0}$ & & intrinsic permeability & \multicolumn{3}{|c|}{$4 \times 10^{-9}$} & \multicolumn{3}{|c|}{$4 \times 10^{-7}$} & $\mathrm{~m}^{2}$ \\
\hline$U_{0}$ & & half-spreading rate & 2 & 4 & 8 & 2 & 4 & 8 & $\mathrm{~cm} / \mathrm{yr}$ \\
\hline$\phi_{0}$ & $\left(\frac{\rho_{s} F_{\max } U_{0} \mu_{0}}{\rho_{f} \Delta \rho K_{0}}\right)^{1 / n}$ & reference background porosity & 2.0 & 2.5 & 3.1 & 0.4 & 0.5 & 0.6 & $\%$ \\
\hline$w_{0}$ & $\frac{\rho_{s} U_{0} F_{\max }}{\rho_{f} \phi_{0}}$ & reference melt velocity & 24.0 & 38.0 & 60.4 & 111.2 & 176.5 & 280.2 & $\mathrm{~cm} / \mathrm{yr}$ \\
\hline$w_{0} / U_{0}$ & & "mobility" & 12.0 & 9.5 & 7.55 & 55.6 & 44.1 & 35.0 & - \\
\hline$\delta_{0}$ & $\sqrt[n]{\frac{K_{0} \phi_{0}^{n} \eta_{0}}{\mu_{0} \phi_{0}^{m}}}=\sqrt[n]{\frac{\eta_{0} w_{0} \phi_{0}}{\Delta \rho g \phi_{0}^{m}}}$ & reference compaction length & 3.9 & 4.9 & 6.2 & 8.4 & 10.6 & 13.4 & $\mathrm{~km}$ \\
\hline$P e$ & $h w_{0} / \kappa_{0}$ & Peclet number & 1044 & 1658 & 2631 & 4846 & 7693 & 12213 & - \\
\hline$R_{f}$ & & freezing rate constant & 100 & 200 & 400 & 100 & 200 & 400 & - \\
\hline
\end{tabular}

207

208

209

$$
\left(\frac{\rho_{f}}{\rho_{s}} \phi_{0} \phi+\left(1-\phi_{0} \phi\right)\right) \frac{\partial T}{\partial t}+\frac{\rho_{f}}{\rho_{s}} \phi_{0} \phi \mathbf{v}_{\mathbf{f}} \cdot \nabla T+\left(1-\phi_{0} \phi\right) \mathbf{v}_{\mathbf{s}} \cdot \nabla T+\beta T \mathbf{v}_{\mathbf{s}} \cdot \hat{\mathbf{k}}+\phi_{0} L_{c p} T \Gamma-\frac{1}{P e} \nabla^{2} T=0
$$

flow formulation including conservation of energy:

$$
\frac{\partial \phi}{\partial t}+\mathbf{v}_{\mathbf{s}} \cdot \nabla \phi-\frac{h^{2}}{\delta_{0}^{2}} \frac{\mathcal{P}}{\zeta}=\Gamma
$$

$$
\frac{h^{2}}{\delta_{0}^{2}} \frac{\mathcal{P}}{\zeta}-\nabla \cdot \frac{\phi^{n}}{\mu}\left[\nabla\left(\mathcal{P}+p^{*}\right)+\hat{\mathbf{k}}\right]=\frac{\Delta \rho}{\rho_{f}} \Gamma
$$

where we solve for the porosity or volume fraction of melt, $\phi$, the compaction pressure, $\mathcal{P}$, and the temperature, $T$. $\zeta$ is the non-dimensional bulk viscosity given by:

$$
\zeta=\frac{\eta}{\left(\phi+\phi_{\epsilon}\right)^{m}}
$$

where an inverse dependence on porosity for $\zeta$ was previously suggested based on homogenization theory (Simpson et al., 2010a) and $m$ is the exponent on porosity. In this formulation of bulk viscosity, a small regularization of porosity, $\phi_{\epsilon}$, is used to avoid singularity in the limit of $\phi \rightarrow 0$.

$\Gamma$ is the non-dimensional interphase mass exchange rate given by:

$$
\Gamma=\Gamma_{+}+\Gamma_{-}
$$


225

where $\Gamma_{+}$is the melting rate:

$$
\Gamma_{+}=G(F) \frac{\left(1-\phi_{0} \phi\right)}{\phi_{0}} \frac{D_{s} f}{D t}
$$

and $\Gamma_{-}$is the freezing rate:

$$
\Gamma_{-}=\frac{1}{2} \phi R_{f}\left[T-T_{\text {liquidus }}^{\text {basalt }}-\left|T-T_{\text {liquidus }}^{\text {basalt }}\right|\right]
$$

$f(T, P)=\left[\frac{T-T_{\text {solidus }}}{T_{\text {liquidus }}-T_{\text {solidus }}}\right]^{\beta_{f}}$ is the volume fraction of melt predicted in a closed system (batch melting) as a function of pressure and temperature, based on a power law parameterization of the peridotite phase diagram (Katz et al., 2003). The peridotite solidus and lherzolite liquidus are defined as functions of the lithostatic pressure (Hirschmann, 2000):

$$
T_{\text {solidus }}=A_{1}+A_{2} P_{L}+A_{2} P_{L}^{2}
$$

$$
T_{\text {liquidus }}=B_{1}+B_{2} P_{L}+B_{2} P_{L}^{2}
$$

where $A_{i}, B_{i}$ are constants (see Table 1$) . \mathrm{G}(\mathrm{F})$ is a tanh function of the local degree of depletion, $F$, that simply ceases melting with clinopyroxene exhaustion. The local degree of depletion, $F$, is calculated by tracking the concentration of a completely compatible trace element, i.e an element with bulk partition coefficient $D \rightarrow \infty$, (Spiegelman, 1996). $R_{f}$ is the freezing rate constant that we vary proportionally with the spreading rate such that melt is kept out of the cold, strong lithosphere. If $R_{f}$ is taken to be zero, there will be no freezing. $T_{\text {liquidus }}^{\text {basalt }}$ is the basaltic liquidus (Hirschmann, 2000) taken to be the same as the peridotite solidus, $T_{\text {solidus }}$.

Permeability is typically described by $K=\frac{a^{2} \phi^{n}}{b}$, where $a$ is the mean grain size or reference spacing of melt rich veins, $b$ and $n$ are empirical constants 
that are constrained experimentally (Zhu et al., 1995; Wark and Watson, 1998). We follow Katz (2008) in our model formulation for dimensional permeability:

$$
K=K_{0} \phi^{n}
$$

where the mean grain size, $a$, and empirical constant, $b$, are incorporated into $K_{0}$, the intrinsic permeability, which is one of the variables we vary in the models (Table 2). The intrinsic permeability has units of $\mathrm{m}^{2}$. Nondimensional permeability is described by

$$
K=\phi^{n}
$$

where $n$ is an empirical constant, constrained experimentally to be between 2 and 3 (Zhu et al., 1995; Wark and Watson, 1998; Miller et al., 2014). Nondimensional liquid viscosity, $\mu$, is taken here to be constant 1.

\subsection{Boundary and initial conditions}

Boundary and initial conditions for these models are shown schematically in Figure 1. Mantle upwelling is driven by imposing ridge-like plate motion along the top boundary such that $U_{\text {top }}=U_{0} \operatorname{erf}\left(\frac{x}{x_{\lambda}}\right)$, where $U_{0}$ is the halfspreading rate, $x$ is the distance from the ridge axis, and $x_{\lambda}=0.01(1 \mathrm{~km})$ is the width of the smoothed step function, which is chosen to represent roughly the width of the neo-volcanic zone at the ridge axis. We allow solid inflow at the bottom of the domain and outflow at the sides such that solid flow tangent to the sides and bottom boundaries is zero (i.e $\mathbf{v}_{\mathbf{s}} \cdot \hat{\mathbf{t}}=0$, where $\hat{\mathbf{t}}$ is the unit vector tangent to the boundary). The top boundary allows melt to outflow but prevents any outflow of the solid mantle (i.e $\mathbf{v}_{\mathbf{s}} \cdot \hat{\mathbf{n}}=0$, where $\hat{\mathbf{n}}$ is the unit vector normal to the boundary). 
The top of the domain represents the Moho, where we apply a Robin condition on the temperature:

$$
\nabla T \cdot \hat{\mathbf{n}}=\frac{T_{s}-T}{h_{c}}
$$

This assumes a linear relationship between ocean surface temperature, $T_{s}=$ $0^{\circ} \mathrm{C}$, and temperature at the Moho over $h_{c}=7 \mathrm{~km}$, the crustal thickness. The temperature solution for the solid system for each model (Figure 2) is used as the initial condition for the two-phase system. Obtaining the compaction pressure involves solving for an elliptic equation that requires boundary conditions on all boundaries. We enforce that the compaction pressure is zero on the bottom boundary before melting, consistent with the fact that solid flow is incompressible outside of the melting regime (i.e. $\nabla \cdot \mathbf{v}_{\mathbf{s}}=0$ implying $\mathcal{P}=0$ ). The top and sides boundaries are Neumann boundaries on the melt flux such that $\nabla \mathcal{P}$ has some value (Equation 4).

The initial condition for porosity consists of two parts:

$$
\left.\phi\right|_{t=0}=\Gamma_{\text {Stokes }} \Delta t+\phi_{\text {tr }}
$$

$\Gamma_{\text {Stokes }}$ is the melt production rate in the solid system, which is solved using the steady state version of Equation 20, such that only the advective part of the material derivative is used and the tanh function $\mathrm{G}(\mathrm{F})$ is omitted. $\Delta t$ is the time step used as an approximation to obtain the initial porosity in the melting region. $\phi_{\text {tr }}$ is an initial porous region to allow melt flow through the axial region during the initial part of these calculations:

$$
\phi_{\operatorname{tr}}=\frac{1}{2} e^{-\left(\frac{x}{-\left(1+\theta_{\operatorname{tr}} z\right) x_{\text {widh }}}\right)^{2}}\left[\tanh \left(\frac{z-z_{0}}{z_{\lambda}}\right)-\tanh \left(\frac{2\left(z-z_{1}\right)}{z_{\lambda}}\right)\right]
$$

where $\theta_{\text {tr }}, x_{\text {width }}, z_{0}, z_{1}$, and $z_{\lambda}$ define the shape of the initial porosity transient and are constants given in Table 1. 

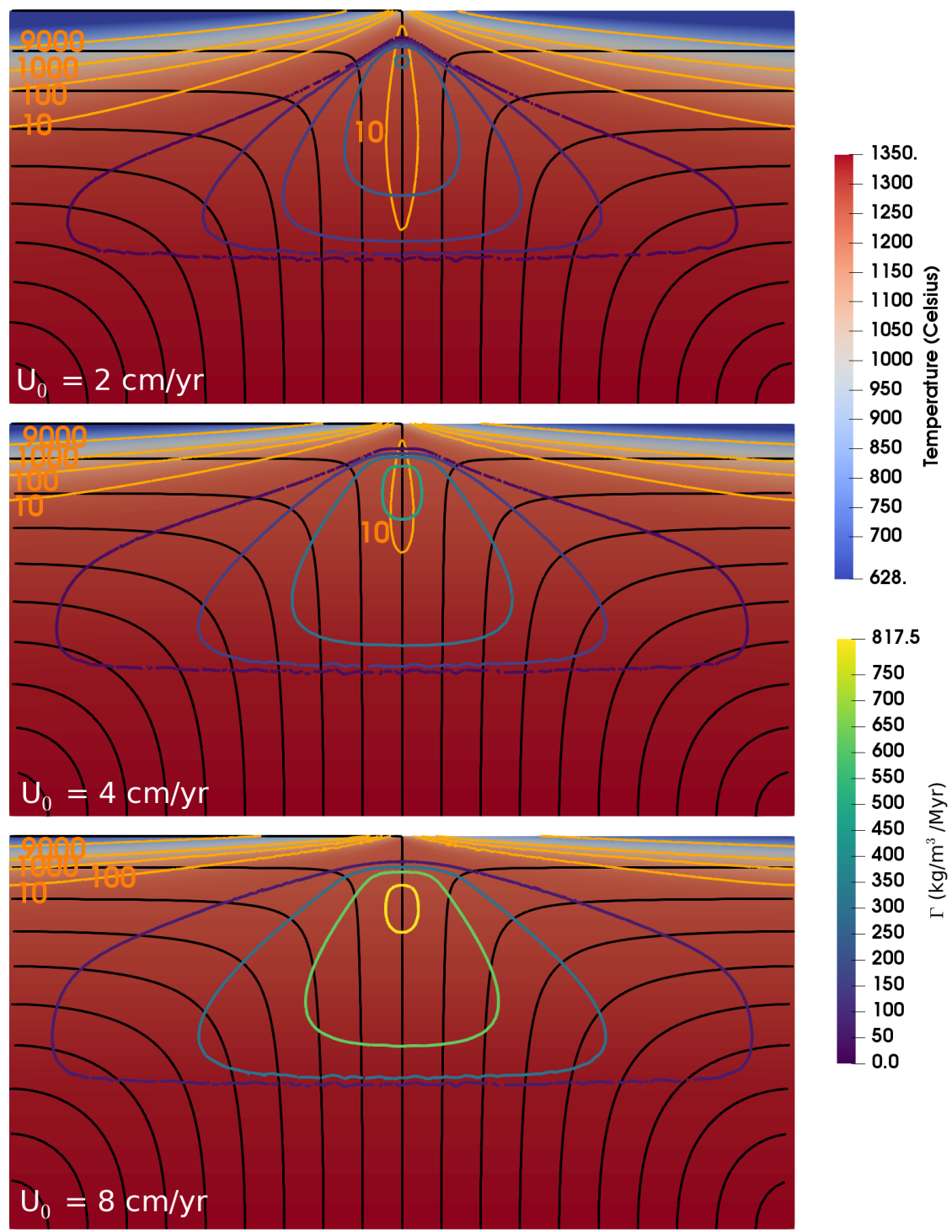

Figure 2: The temperature solutions from the solid system for half-spreading rates $U_{0}=$ $2,4,8 \mathrm{~cm} / \mathrm{yr}$. Black lines are solid flow lines. Yellow to purple contours are melting rate production, $\Gamma$. Orange labeled contours are non-dimensional viscosity contours scaled by $\eta_{0}=10^{19} \mathrm{~Pa} \mathrm{~s}$. 


\subsection{Numerical implementations}

We explore the two-phase dynamics for mid-oceanic ridges by varying half-spreading rates $U_{0}=2,4,8 \mathrm{~cm} / \mathrm{yr}$ and intrinsic permeability, $K_{0}=$ $4 \times 10^{-7}, 10^{-9} \mathrm{~m}^{2}$. We discretize the non-dimensionalized set of equations using finite differences in time and finite elements in space. Dynamic pressure is chosen to be a piecewise linear function (P1) while temperature, solid velocity and compaction pressure are piecewise quadratic (P2). Porosity is discontinuous piecewise quadratic (P2DG). We use TerraFERMA, the Transparent Finite Element Rapid Model Assembler (Wilson et al., 2017) to construct and solve our non-dimensional, nonlinear system of equations in two dimensions. TerraFERMA leverages open source libraries, FEniCs (Logg et al., 2011, 2012), PETSc (Balay et al., 2017) and SPuD (Ham et al., 2009) to provide a common interface for building custom finite element method models that are transparent and reproducible. We use Gmsh (Geuzaine and Remacle, 2009) to generate an unstructured triangular mesh over the rectangular domain. The smallest element is $\sim 100 \mathrm{~m}$ at the ridge axis and coarsens away from it, with the largest element being $\sim 8 \mathrm{~km}$.

The flexibility of TerraFERMA allows us to build on this current model and expand it to be fully coupled or test other parameterizations. This flexibility is an important design feature of TerraFERMA and other software such as ComSol (www.comsol.com) and Underworld2 (Moresi et al., 2007). For these types of complex highly coupled problems, the flexibility gives it an advantage over other software that can be purpose specific and therefore difficult to modify. An example of fully coupled ridge model with an isoviscous rheology is included in Wilson et al. (2017). This suite of models are openly 
available to the readers in the form of TerraFERMA markup files (.tfml) at https://github.com/joycesim/M3LT_one_Uall.git.

\section{Results}

The solid Stokes system represents passively driven single phase flow with thermal feedback. The temperature solutions from the solid system for all the spreading rates show the cooling - hence strengthening - and thickening of the oceanic lithosphere with distance away from the ridge axis (Figure 2). The solid mantle velocity field resembles a typical corner flow (black flow lines in Figure 2). Most of the melt is generated on the ridge axis, where the solid mantle is upwelling at the fastest rates, although the melting region (colored contours in Figure 2) is as wide as the width of the domain. The melting rate is higher for faster spreading rates due to faster upwelling rates.

In the two-phase system, these models are initialized with the solid velocity, dynamic pressure and temperature from the solid system with corresponding spreading rate (Figure 2) and initial conditions for porosity and compaction pressure as described in the previous section. The models go through an initial transient period before settling into a quasi-steady state (Figure 3). During the transient phase, large amplitude porosity waves propagate from the melting region through the relatively low porosity region between the top of the melting region and the moho. Porosity waves are a natural consequences of poro-viscous flow with non-zero compaction length (Spiegelman, 1993b). In these models, the porosity waves are transient features except in the high permeability, low spreading rate case, in which the waves persist throughout the model run. These high amplitude porosity 
waves occur when the melt flux varies on length scales comparable to the compaction length:

$$
\delta=\sqrt{\frac{K \phi^{n} \eta}{\mu \phi^{m}}}
$$

and they are dependent on the initial conditions. The reference values for compaction length for all model runs are given in Table 2. At quasi-steady state, there are two main melt transport patterns. Near the ridge axis, melt moves horizontally towards the ridge axis to form a central high porosity region, which eventually moves out of the domain at the top of the ridge axis. Away from the ridge axis, buoyant melt rises up to encounter the cold, and thus stronger, sloping lithosphere, where it is either diverted to the ridge axis or freezes into the lithosphere.

\subsection{Crustal production versus spreading rate}

The variation of oceanic crustal thickness with spreading rate is a first order seismically observable feature of mid-ocean ridges (White et al., 1992). In order to validate the models with seismic observations, we use three model output proxies for crustal thickness: 1) total melt production rate in the purely solid Stokes system ignoring the effect of melt transport, 2) total melt production rate in the two-phase system and 3) melt flux through the top boundary. Using mass balance, the total amount of melt produced gives an upper bound of the amount of oceanic crust that can be produced:

$$
2 \rho_{c} h_{c} U_{0}=\rho_{s} \phi_{0} w_{0} h \int \Gamma^{*} d X
$$

where $h_{c}$ is the oceanic crustal thickness, $\rho_{c}$ is the oceanic crustal density assumed to be $2800 \mathrm{~kg} / \mathrm{m}^{3}, \int \Gamma^{*} d X$ is the non-dimensional total melt production rate in the whole domain $(d X)$ either in the solid system, $\Gamma^{*}=\Gamma_{\text {Stokes }}$, 


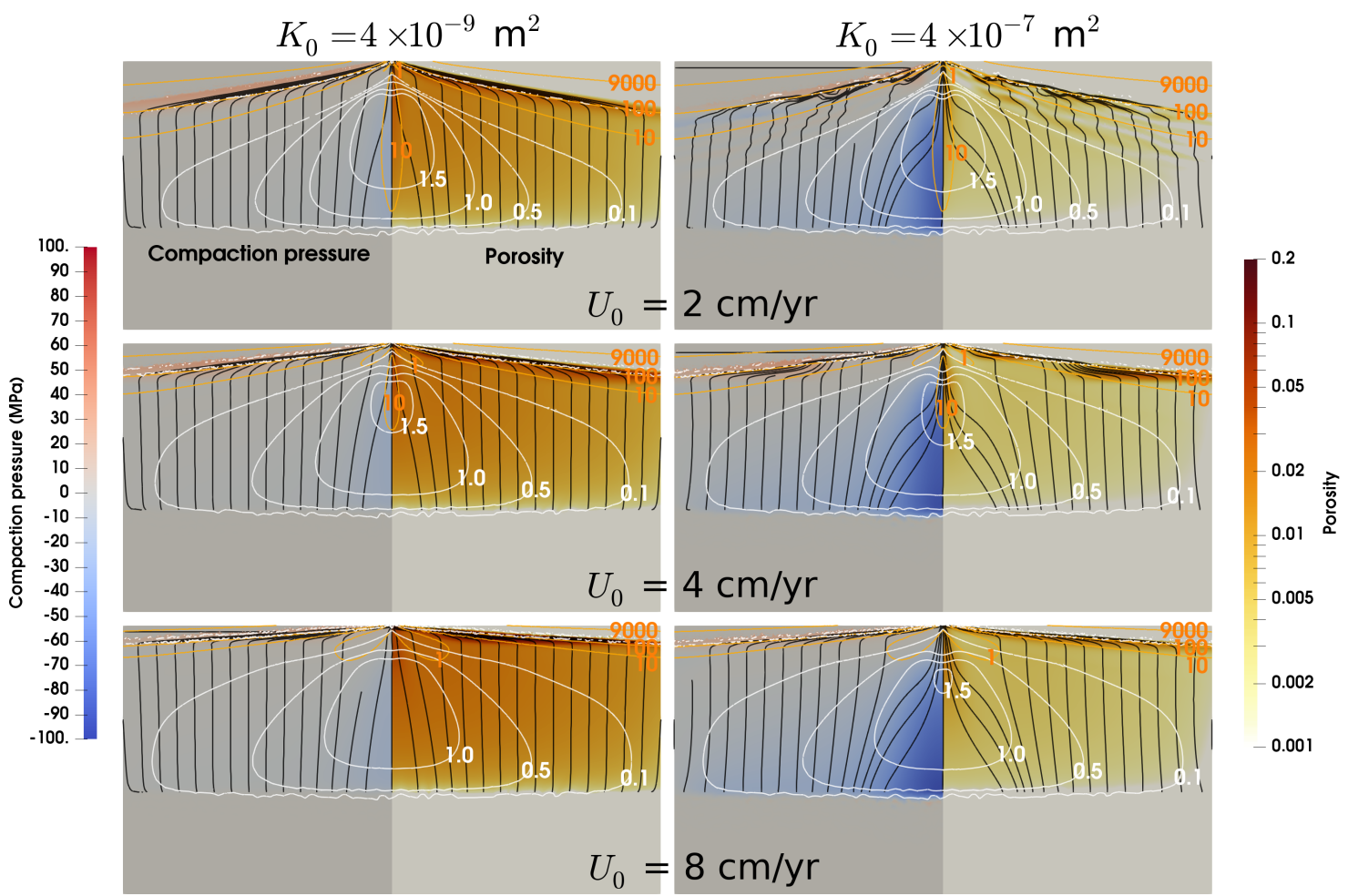

Figure 3: Snapshots of the dimensional compaction pressure and porosity field at the end of all six model runs with increasing permeabilities to the right and increasing halfspreading rates down the panels. Domain of each panel is $200 \mathrm{~km}$ wide and $100 \mathrm{~km}$ deep. The left side of each panel shows the dimensional compaction pressure. The right side of each panel shows the porosity (i.e. $\phi=0.01$ is $1 \%$ porosity). The black lines on both sides of the each panel track the melt flow. White contours are for non-dimensional total melting rate, $\Gamma$. 
or, in our second estimate, the two-phase system, $\Gamma^{*}=\Gamma_{+}$. The mass balance for oceanic crustal production based on total melt flux through the top boundary in the two-phase system is:

$$
2 \rho_{c} h_{c} U_{0}=\rho_{f} \phi_{0} w_{0} h \int \mathbf{q} \cdot \hat{\mathbf{n}} d s_{t o p}
$$

where $\hat{\mathbf{n}}$ is the unit outward normal to the top boundary, $d s_{\text {top }}$, and $\mathbf{q}=\phi \mathbf{v}_{\mathbf{f}}$ is the melt flux.

The melt system is time-dependent, causing fluctuations in both melt production rate and melt flux at the ridge axis (Figure 4a). The crustal thickness calculated using the total melt production rate for the two-phase models are similar, regardless of permeability, whereas those calculated by integrating the melt flux through the top boundary are smaller. After an initial transient of about $\sim 2$ Myrs, however, the system settles into a quasisteady state and we calculate mean melt production rates and melt fluxes over this period (Figure 4b). Only the crustal thickness estimates using melt flux from model with large permeability and slowest spreading has persistent fluctuations and this is due to the sustained porosity waves through the model run (top right panel in Figure 3).

Crustal thicknesses from both model estimates and geophysical observations are plotted together as a function of spreading rate (Figure $4 \mathrm{~b}$ ). The crustal thickness predicted from the total melt production rate for the twophase models are slightly larger than those from the solid model because there is more melting in the two-phase model due to a warmer mantle on the ridge axis from melt advection. Some of the melt produced freezes back into the lithosphere while most of the melt leaves the top boundary and is interpreted here as forming oceanic crust. There is more freezing in the models 
375 with smaller permeability, particularly for faster spreading rates, as shown 376 by the smaller amount of crustal thickness predicted from melt flux through 377 the top boundary (Figure $4 \mathrm{~b}$ ). 


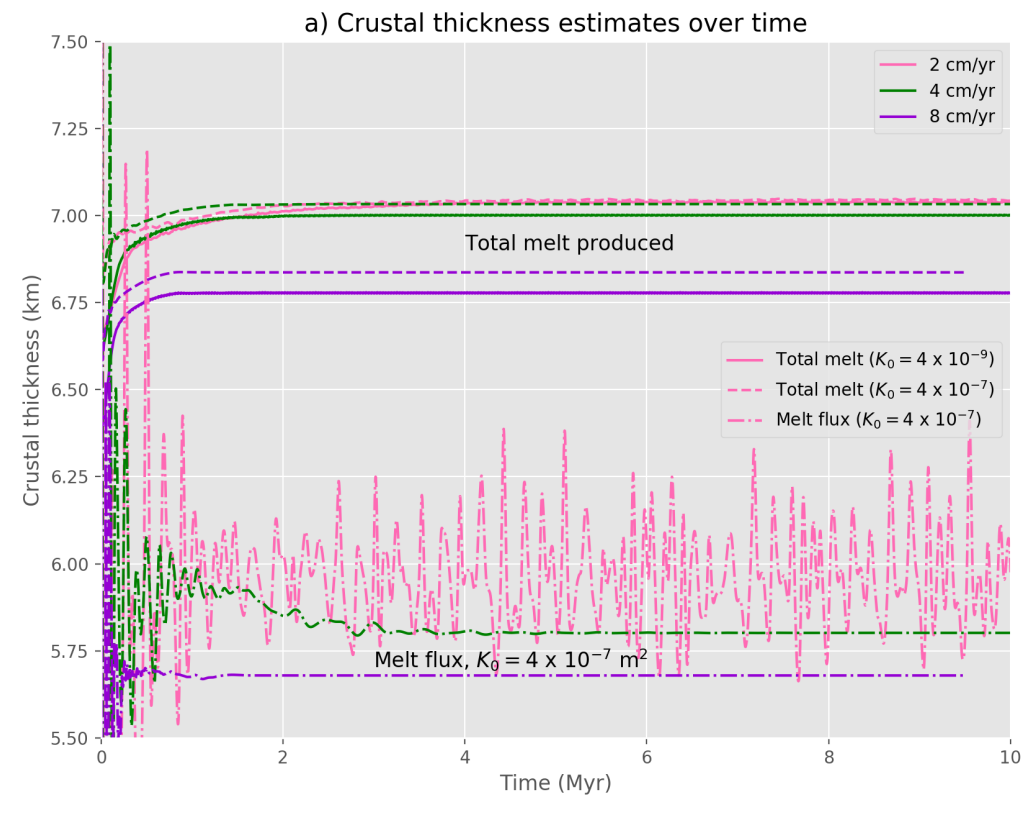

b) Crustal thickness versus half spreading rate

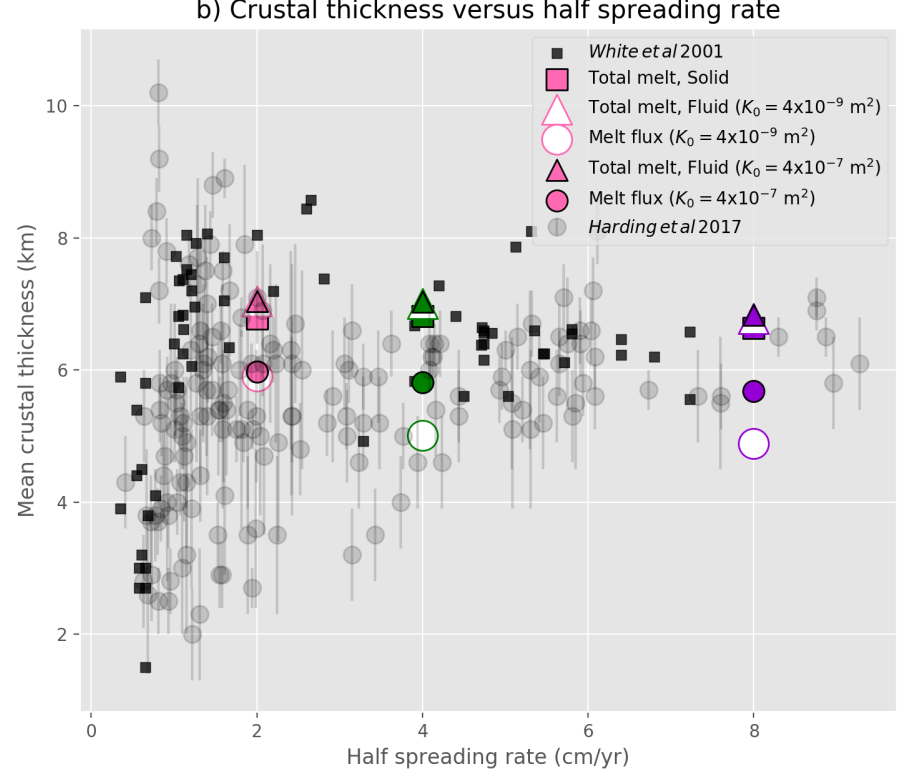

Figure 4: Model predictions of crustal thicknesses for all models compared with geophysical observations. a) Crustal thickness calculated for each model as a function of time. Crustal thickness is calculated using two different methods. 
Figure 4 (continued): Solid and dashed lines represent crustal thickness predictions from total melt produced in models with smaller and larger permeability respectively. Dashdotted lines represent crustal thickness predictions from melt flux for models with larger permeability. b) A plot of mean crustal thickness production predicted versus halfspreading rate, $U_{0}$, for the models presented in this work along with geophysical observations (White et al., 2001; Harding et al., 2017). Colored squares are the crustal thicknesses calculated from the total melt produced in the solid system, ignoring melt transport. Triangles represent the mean crustal thicknesses predicted from the time series of total melt production rate for the two-phase system (solid and dashed lines in panel a) after the transient $\sim 2$ Myrs. Circles represent the mean crustal thicknesses predicted from the time series of the melt flux coming out of the top boundary of the model domain (dash-dotted lines in panel a). Filled and unfilled triangles and circles represents model predictions for models with lower and higher permeability respectively. The unfilled triangles are covered by the filled triangles since they are almost always nearly identical. Black squares are crustal thickness inferred from an active seismic data compilation (White et al., 2001). Grey circles are from a more recent compilation of active seismic data along with error bars (Harding et al., 2017).

Along with our mean estimates from our models in Figure 4b, a compilation of observed oceanic crustal thicknesses versus spreading rates from active seismic surveys is plotted in black squares (White et al., 1992; Bown and White, 1994; White et al., 2001) along with a more recent compilation plotted in grey circles (Harding et al., 2017). The older compilation of seismic data suggests that crustal thickness is about $7 \mathrm{~km}$ independent of half-spreading rate for half-spreading rate more than $1 \mathrm{~cm} / \mathrm{yr}$ and rapidly decreases for slower spreading rates. The more recent compilation of Harding et al. (2017) gives an average oceanic crustal thickness of $\sim 6 \mathrm{~km}$. The recent compilation also suggests that not only does the oceanic crustal thick- 
nesses vary more than previously observed, this variability seems to decrease as spreading rates increase. Crustal thickness estimates from our model outputs agree well with the seismic observations for half-spreading rates more than $2 \mathrm{~cm} / \mathrm{yr}$, thus giving some confidence in the robustness of the models. It also suggest that fluctuations in melt flux due to porosity waves could contribute to the variable crustal thickness at slower spreading rate.

\subsection{Importance of permeability}

The permeability of the solid mantle matrix determines how quickly melt generated can segregate from the solid mantle and controls the relative importance of solid advection and pressure driven flow. For example, equation 4 shows that if the solid were impermeable, melt could not separate from the solid and would solely be advected by the solid phase (and the porosity would equal the degree of melting, i.e. $\phi \sim F)$. Large $K_{0}$ implies that the melt flow is more dominated by pressure gradients than solid advection. Since the total melt production rate is bounded by the solid flow field, increasing the intrinsic permeability also means that less melt can be retained in the mantle, i.e. higher intrinsic permeability, $K_{0}$, leads to a faster reference melt velocity, $w_{0}$, and lower amount of background melt or porosity, $\phi_{0}$ (Table 2). Figure 3 shows that the maximum porosity for high intrinsic permeability ( $K_{0}=10^{-7}$ ) is $\sim 2 \%$ versus $20 \%$ for lower $K_{0}$ while both models have the same melt production rate (Figure 2). There are two main regions of higher porosity accumulation, at depths on the ridge axis and off to the sides beneath the LAB, which is defined by the temperature dependent rheological and freezing boundary. The accumulation of melt beneath the LAB in these models is consistent with the "decompaction melt layers" described initially 
by Sparks and Parmentier (1991).

The intrinsic permeability alters melt transport patterns. At higher intrinsic permeability, a wider region of melt near the ridge axis feeds the central high porosity region (Figure 3) with more pronounced horizontal melt transport. This is coupled with larger magnitude and therefore gradient of compaction pressure (Figure 3), which we will discuss further in the following section. The "mobility", $w_{0} / U_{0}$, is the ratio of the reference melt velocity to the spreading rate and is a measure of how strongly melt transport deviates from solid mantle flow. If no melt segregation occurs, or $w_{0} / U_{0}=1$, melt will simply follow the solid mantle flow. For lower intrinsic permeability, the mobility is smaller (Table 2), suggesting that the melt deviates less from the solid mantle flow compared to models with larger intrinsic permeability, and larger mobility (Figure 3). The largest mobility is in the model case with slow spreading and high intrinsic permeability, where porosity waves are persistent.

\subsection{Melt transport due to pressure gradients}

Melt focuses due to horizontal pressure gradients. In the model formulation for this study, pressure is decomposed into 1) compaction pressure, 2) dynamic pressure and 3) lithostatic pressure as shown in Equation 1. The horizontal gradient of dynamic pressure, $p^{*}$, is only significant near the ridge axis (first column in Figure 5). The length scale over which this pressure exerts itself is a balance between pressure gradients induced by incompressible shear and those due to melt buoyancy in the vicinity of the ridge axis 
436

(Spiegelman and McKenzie, 1987):

$$
L_{\text {ridge }} \propto \sqrt{\frac{\eta U_{0}}{\Delta \rho g}}
$$

437

$L_{\text {ridge }}$ are small $(\sim 1-2 \mathrm{~km})$ in the models presented here.

Compaction pressure, $\mathcal{P}$, is largest in magnitude where the solid mantle begins to melt above the dry peridotite solidus on the ridge axis. When compaction pressure is positive, the solid matrix is expanding or pore spaces are opening up. One can also think of it as an overpressure of the liquid phase with respect to the solid phase. When compaction pressure is negative, the solid matrix is contracting or pore spaces are contracting. Here, there is an underpressure of the liquid phase with respect to the solid phase. Horizontal compaction pressure gradient is comparable to buoyancy within the melting region and along the decompaction layers (second and third columns in Figure 5). The increase in intrinsic permeability between model runs correlates with an order of magnitude increase in horizontal compaction pressure gradient. Spreading rate does not change the magnitude of the pressure gradients. Away from the ridge axis where the horizontal compaction pressure is larger, melt flow is vertical due to buoyancy. The horizontal compaction pressure gradient is also significant where the decompaction layers are, pushing melt away from the cold and therefore strong lithosphere, thus focusing melt towards the ridge axis. Bands of positive and negative horizontal compaction pressure gradients are present where there are porosity waves in the model run with slowest half-spreading rate and larger intrinsic permeability (top right in Figure 5). 


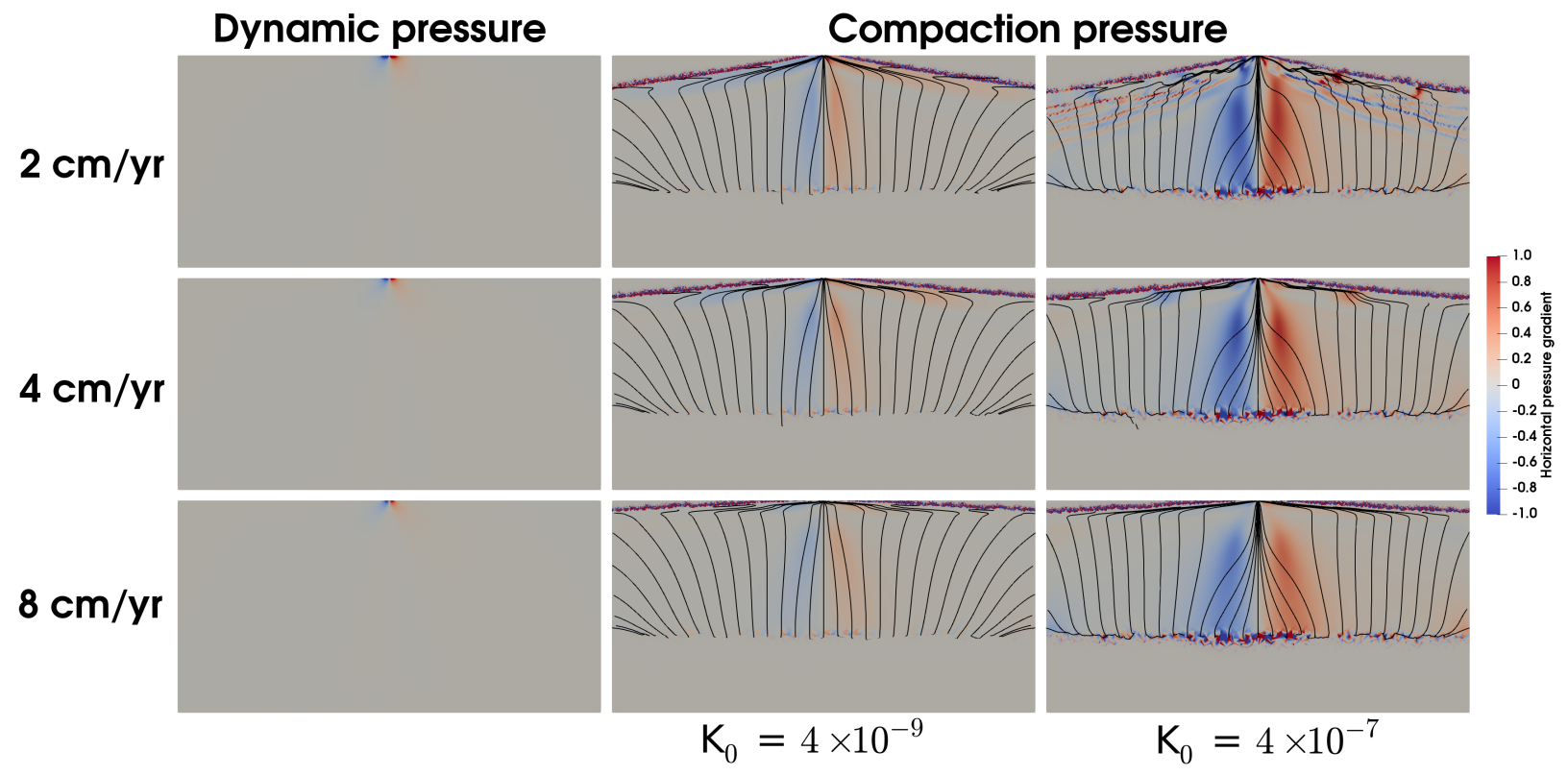

Figure 5: Comparison of non-dimensionalized horizontal pressure gradients across all models. All pressures are non-dimensionalized using $\Delta \rho g h \sim 500 \mathrm{MPa}$.

\section{Discussion}

\subsection{Melt focusing}

There are three melt focusing mechanisms that we identify in our models:

1) Melting pressure focusing, 2) Decompaction layers and 3) Ridge suction (Figure 6). Melting pressure focusing is a dominant feature in these models that was recently recognized (Turner et al., 2017). In these models, melt generated deep in the melting region near the ridge axis is focused towards the ridge axis (Figure 3) due to the horizontal compaction pressure gradient (Figure 5). Turner et al. (2017) discusses this effect but also explored how grain growth evolution and grain size dependent viscosity affects melt dynamics in a two-phase flow model applied to mid-ocean ridges. We want 
to emphasize that this mechanism is robust. In our models, melting pressure focusing is observed with a variable viscosity without any grain size dependence and we will show that it can develop even in an isoviscous model with sufficiently large shear viscosity.

In quasi-steady state in the frame of the solid and with the small porosity approximation, the dimensional conservation of mass for porosity dictates that the volumetric strain-rate of the solid (compaction rate) balances melt production rate:

$$
\nabla \cdot \mathbf{v}_{\mathbf{s}} \approx-\frac{\Gamma}{\rho_{s}}
$$

This balance assumes that melt segregation is efficient and that the solid advection of melt is negligible, which is similar to Turner et al. (2017). Because the compaction pressure is related to the volumetric strain rate, i.e. $\mathcal{P}=\zeta \nabla \cdot \mathbf{v}_{\mathbf{s}}$ (Equation 2), it follows that in quasi-steady state,

$$
\mathcal{P} \approx-\zeta \frac{\Gamma}{\rho_{s}}
$$

Therefore, the compaction pressure assumes the shape of the total interphase exchange rate field, $\Gamma$, with amplitude controlled by the bulk viscosity, $\zeta$. For adiabatic melting beneath ridges, $\Gamma$ is always roughly triangular shaped with a maximum melting rate on the ridge axis, and therefore, a minimum compaction pressure on axis (Figure 3). The bulk viscosity, $\zeta$, depends on the shear viscosity, $\eta$, and is inversely proportionate to the porosity, $\phi$, since the bulk viscosity formulation used in this work is $\zeta \sim \frac{\eta}{\phi}$ (Equation 18). The bulk viscosity can be significantly larger than the shear viscosity for small porosities. In particular, higher permeability systems should show greater melting pressure focusing effects for the same shear viscosity, $\eta$, since higher permeabilities lead to smaller retained porosities (Figure 5 and Table 2). 
Decompaction layers are melt rich layers along which melt is focused towards the ridge axis (Sparks and Parmentier, 1991; Spiegelman, 1993c; Hebert and Montési, 2010; Keller et al., 2017). Melt generated in the melting region is more buoyant than the surrounding mantle and segregates upwards to the LAB. While melt cannot permeate a rheologically strong lithosphere, it can also begin to freeze as it encounters a colder lithosphere, making an impermeable layer. The compaction overpressure opens up pore space below this impermeable layer, thus forming the decompaction layers that deflect melt towards the ridge axis. The balance of melt deflection versus freezing is delicate and depends on both the rheological and freezing rate parameterization (Spiegelman, 1993c). Decompaction channels can be present even in an isoviscous case as long as freezing occurs on a length scale comparable to the compaction length scale (Spiegelman, 1993c) as given in Equation 29, which depends on the rheological models. The cold and therefore strong lithosphere increases the compaction length scale. Turner et al. (2017) have decompaction layers that are less prominent on the freezing front, since it is below the rheologically stronger lithosphere, which would make the compaction length larger.

As tectonic forces pull oceanic plates apart, the dynamic pressure draws melt towards the ridge axis from the surrounding region due to incompressible shear (Spiegelman and McKenzie, 1987; Morgan, 1987). This ridge suction focusing mechanism depends on spreading rates and shear viscosity (Equation 32) such that the dynamic pressure becomes larger in magnitude as spreading rate increase for the same shear viscosity, $\eta$. However, faster spreading rate leads to a hotter sub-Moho mantle and therefore, lower 


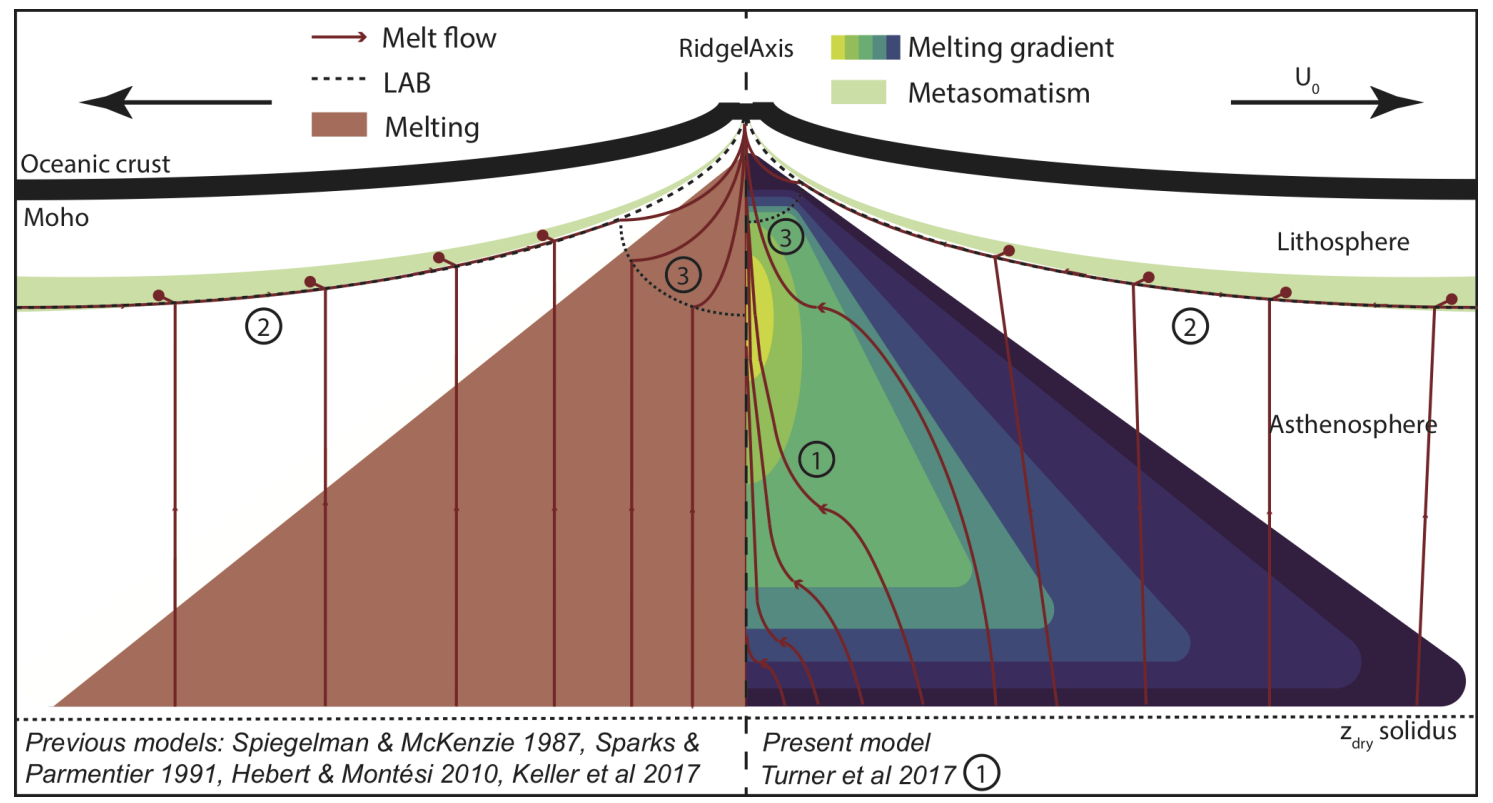

Figure 6: Illustration of melt focusing mechanisms from past and present work based on Keller et al. (2017). The three melt focusing mechanisms are numbered: 1) Melting pressure focusing 2) Decompaction layers and 3) Ridge suction. The dashed black line down the center represents the ridge axis. The thick black curved lines that connect at the highest point at the ridge axis represent the oceanic crust. The Moho is the bottom of the oceanic crust. Modeled or hypothesized melting is represented as the half triangle on the left for previous work while it is represented by a lime green to dark violet melting triangle on the right for these models presented. Red lines and arrows indicate melt flow and direction. Red circles indicate where melt freezes into the lithosphere in the green region of metasomatism above the black dashed line for the lithosphere-asthenosphere boundary (LAB). 
shear viscosity, which balances with spreading rate such that the product $\eta U_{0}$ related to shear stress (Equation 32$)$ actually remains roughly the same regardless of spreading rate. In addition, advection of heat by the melt will weaken the solid in a fully coupled model with temperature-dependent shear viscosity as compared to the one-way coupled models in this work. With a weaker ridge axis, the already small ridge suction focusing effect will be even weaker relative to the other two dominant melt focusing mechanisms.

All the focusing mechanisms illustrated in Figure 6 exist in any two-phase flow model that include pressure gradients due to both incompressible and compressible viscous deformation. However, the different mechanisms may not manifest themselves due to specific model choices for the constitutive rheological relations (i.e shear and bulk viscosities). Melting pressure focusing was generally insignificant and therefore not recognized in previous models because of different model choices for mantle rheology. In particular, Spiegelman and McKenzie (1987) shows the ridge suction effect clearly but would not have this melting pressure focusing effect since their models have no melting and therefore no volumetric deformation. In contrast, the ridge suction effect would be smaller for a smaller background shear viscosity $\left(\eta \sim 10^{19}\right)$ as used in this study.

To understand how melting pressure focusing is affected by rheological choices for shear viscosity, we present five models with different shear viscosity models, for half spreading rate, $U_{0}=4 \mathrm{~cm} / \mathrm{yr}$, and intrinsic permeability, $K_{0}=4 \times 10^{-7} \mathrm{~m}^{2}$ (Figure 7 ). We have three constant shear viscosity models, $\eta=10^{19}, 10^{20}, 10^{21} \mathrm{~Pa} \mathrm{~s}$, with a weak ridge axis $\sim 1 \mathrm{~km}$ radius (Figure $7 \mathrm{a}, \mathrm{b}$ and c). To clarify the role of melting pressure focusing, these models do not 


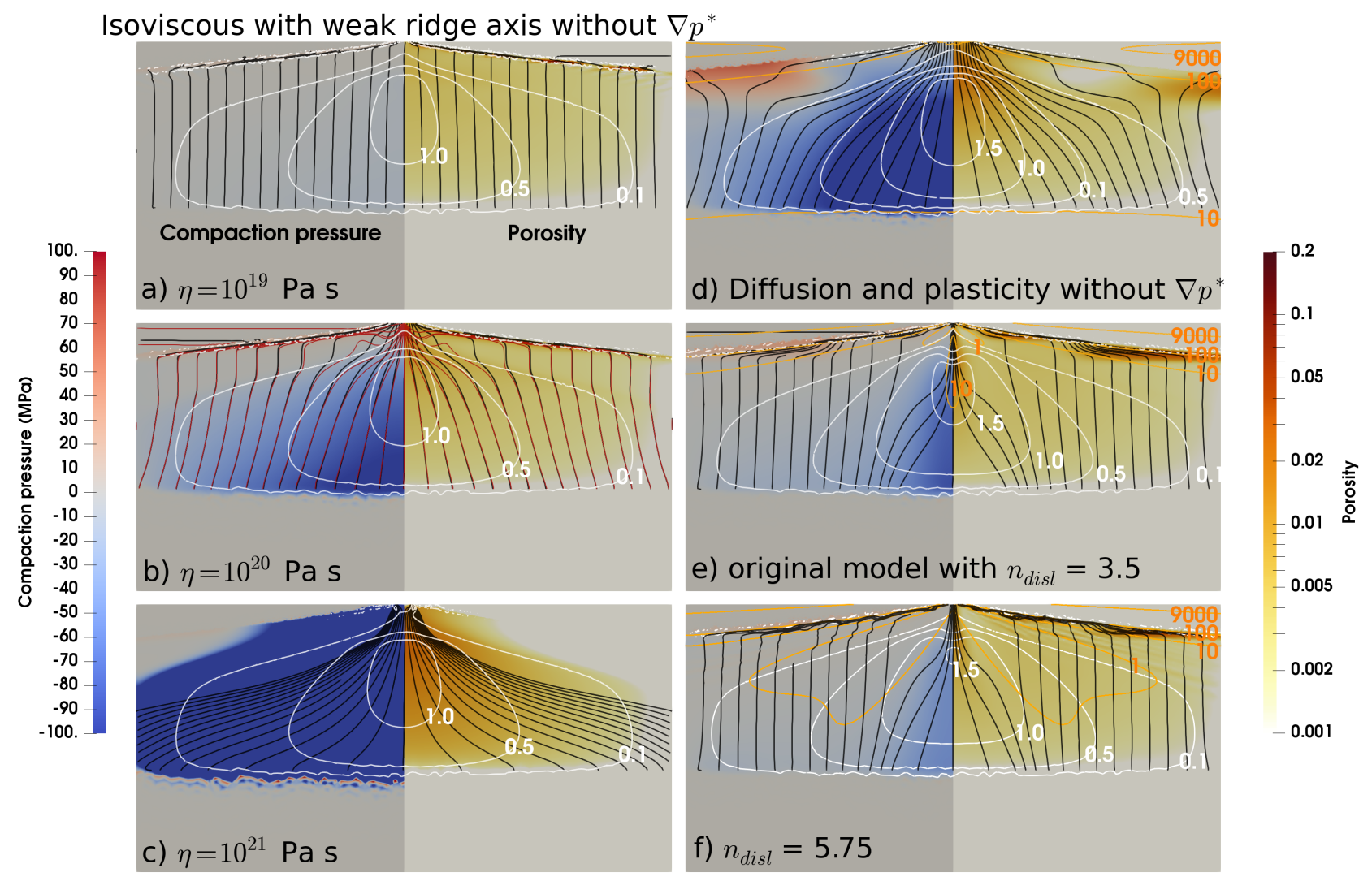

Figure 7: Snapshots of dimensional compaction pressure and porosity at the end of model runs with different rheological choices made. All models have half spreading rate, $U_{0}=4$ $\mathrm{cm} / \mathrm{yr}$, and intrinsic permeability, $K_{0}=4 \times 10^{-7} \mathrm{~m}^{2}$. Domain of each panel is $200 \mathrm{~km}$ wide and $100 \mathrm{~km}$ deep. The left side of each panel shows the compaction pressure in MPa. The right side of each panel shows the porosity (i.e. $\phi=0.01$ is $1 \%$ porosity). The black lines are melt streamlines. The white contours are melting rate contours. The orange contours are the non-dimensional shear viscosity with labels. On the left column, we show three models with increasing constant shear viscosity, $\eta=$ a) $10^{19} \mathrm{~Pa} \mathrm{~s} \mathrm{b)} 10^{20} \mathrm{~Pa} \mathrm{~s}$ and c) $10^{21}$ $\mathrm{Pa}$ s. The isoviscous model cases have a ridge axis weaker by $\sim 100$ orders of magnitude, which is $\sim 1 \mathrm{~km}$ in radius and have no $\nabla p^{*}$ included in Equation 4 . Red lines in panel b) are melt streamline for the model case with grad $\nabla p^{*}$ included. On the right column, we present models with d) diffusion creep and plasticity without $\nabla p^{*}$ included, e) the original model as in Figure 3e) and f) the original model with strain rate exponent $n_{\text {disl }}=5.75$. 
include the the ridge suction effect by neglecting the dynamic pressure $\nabla p^{*}$ in the melt flow field. This effect should only be important with a suction length, $L_{\text {ridge }}$ (Equation 32), near the axis as shown in Figure 7b, which compares melt flows with and without the $\nabla p^{*}$ term in Equation 4 at $\eta=10^{20}$ Pa s. Figure 7 also compares the behavior of the original model (Figure 3) with two more models with composite rheologies. The first just uses a mixture of diffusion creep and plasticity (Equations 12 and 14) without $\nabla p^{*}$ (Figure $7 \mathrm{~d}$ ). The second combines diffusion creep, plasticity and dislocation creep but with a larger stress exponent $n_{\text {disl }}=5.75$ to mimic the effects of grain-size reduction (Figure 7f) as suggested by Turner et al. (2017). The behavior of all of these models can be readily understood given the structure of the compaction pressure (Equation 34). The shape of the pressure field is controlled by the structure of $\Gamma$ which is roughly the same for all models, while the magnitude of the pressure drop depends on choice of bulk viscosity models.

The isoviscous cases clearly illustrate how melting pressure focusing works. All three cases have identical melting rate fields, $\Gamma$. The compaction pressure is primarily controlled by the shear viscosity which is spatially constant. These calculation suggest that the melt pressure focusing becomes important for mean shear viscosities of order $10^{20} \mathrm{~Pa}$ s. At shear viscosities less than $10^{20} \mathrm{~Pa} \mathrm{~s}$ melt pressure focusing is negligible and melt transport is primarily vertical. At higher shear viscosities, the melting pressure focusing effect dominates over buoyancy, causing significant lateral melt transport deep in the melting region. It should be noted that the variation in bulk viscosity in between these isoviscous models is actually smaller than the variation in 
shear viscosity as the porosity is concentrated in specific regions.

As stated, these calculations do not include the ridge suction effect which is expected to only be important within a suction length, $L_{\text {ridge }}$ (Equation 32), around the ridge axis. For $\eta=10^{20} \mathrm{~Pa} \mathrm{~s}, L_{\text {ridge }}$ is small $(\sim 3 \mathrm{~km})$ and ridge suction effect is unimportant (Figure 7b). For shear viscosity, $\eta=10^{21} \mathrm{~Pa} \mathrm{~s}$, $L_{\text {ridge }}$ is larger $(\sim 10 \mathrm{~km})$ and ridge suction effect could contribute to ridge focusing (Spiegelman and McKenzie, 1987).

While the isoviscous model cases serve to help us understand the melting pressure focusing mechanism, models with more realistic rheologies (e.g. presence of lithosphere) help us understand the mechanism in the context of the Earth. The model with diffusion creep and plasticity (Figure 7d) without ridge suction effect, has a mean shear viscosity in the melting region of $\sim 10^{20} \mathrm{~Pa} \mathrm{~s}$ (for this choice of rheological parameters) and shows significant melting pressure focusing in the melting region. Adding dislocation creep mechanisms (Figure 7d and f) weakens the shear viscosity in the regions flanking the ridge axis where the shear strain is greatest. This leads to additional spatial gradients in the compaction pressure which weakens the melting pressure focusing effect. Models with higher powerlaw exponent as suggested by Turner et al. (2017) do not alter the extent of the melting pressure focusing but rather the melt pathways due to the varying strength of horizontal compaction gradients with depth (Figure 7e and f).

The magnitude of the compaction pressure due to melting, depends on the bulk viscosity which is an effective property of the two phase medium. In most early two phase formulations, the bulk viscosity relation was chosen as $\eta / \phi^{m}$ with $m$ as a free parameter usually chosen to be 0 or 1 . In these mod- 
els, we use $m=1$, which was shown to be consistent with the two-phase flow equations using homogenization theory (Simpson et al., 2010a,b). This bulk viscosity formulation, $\zeta \sim \eta / \phi$, amplifies the bulk viscosity since porosities are much smaller than 1, thus affecting the compaction pressure term. Recent work on homogenization of other viscosity mechanisms (Rudge, 2018a,b) provide additional functional relationships between $\zeta, \eta$ and $\phi$, which give a bulk viscosity that is more comparable to the shear viscosity, potentially weakening the melting pressure focusing mechanism. 

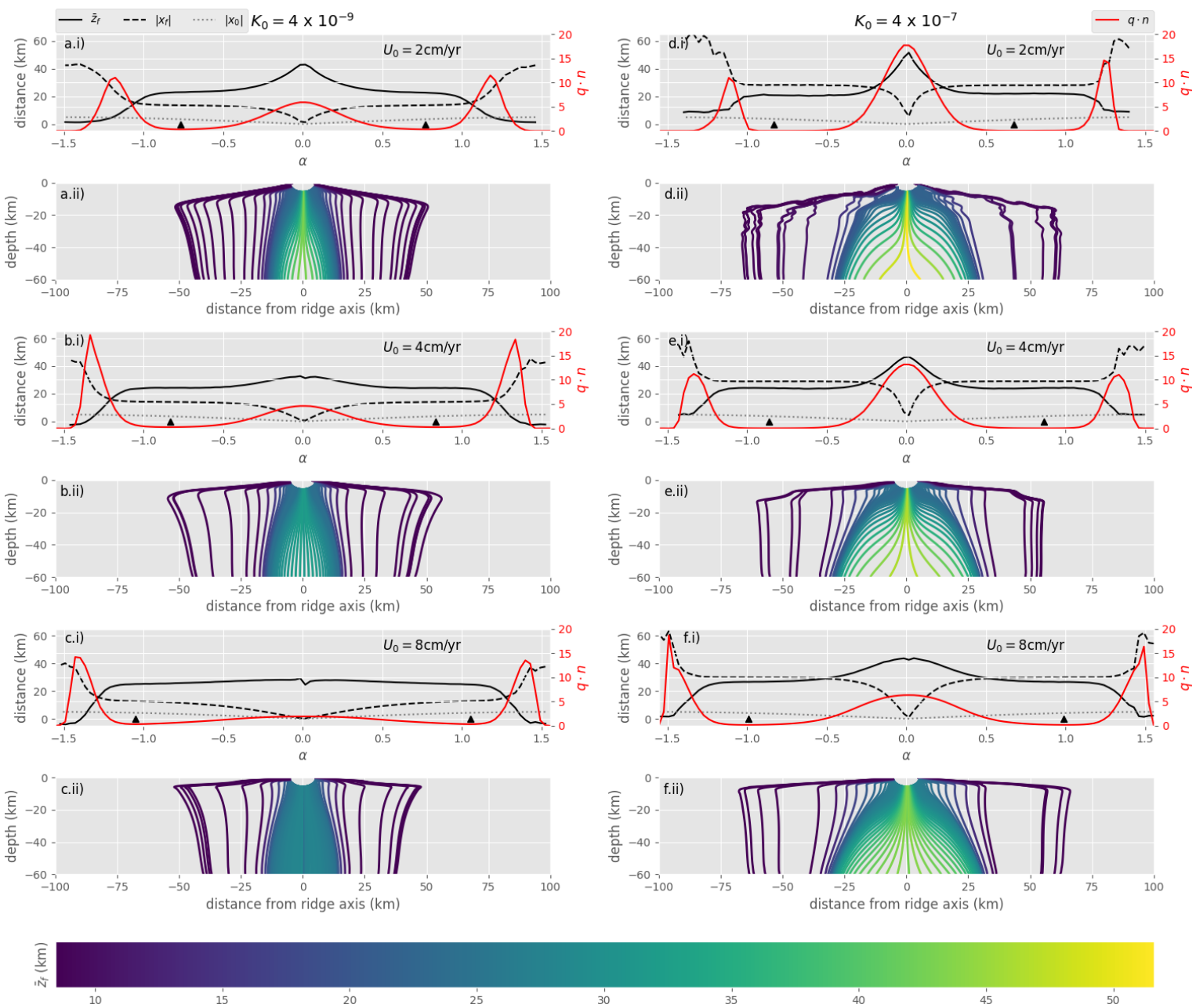

Figure 8: Mean focusing depth, horizontal melt transport distance, non-dimensional melt flux and melt streamlines based on an arc of $5 \mathrm{~km}$ radius centered at the ridge axis at the end of all model runs in Figure 3 with increasing permeabilities to the right and increasing half-spreading rates down the panels. Each model run has two corresponding panels, panels i) with plots of values along the arc and panels ii) showing melt streamlines emanating from the arc. 
Figure 8 (continued): In panels i) of each model set, the black solid line is the mean focusing depth of each streamline, $\bar{z}_{f}$, the black dashed line is the horizontal distance to the origin of melt at the end of each streamline along the arc from the ridge axis, $\left|x_{f}\right|$, the grey dotted line is the horizontal distance to the arc from the ridge axis, $\left|x_{0}\right|$, and the red line is the non-dimensional melt flux normal to the arc. The black triangles represent the locations of the minimum normal melt flux on the arc. The $\mathrm{x}$ and $\mathrm{y}$ axis correspond to distance $(\mathrm{km})$ and radians along the arc. In panels ii) of each model set, melt flux streamlines emanating from the arc of $5 \mathrm{~km}$ radius are plotted along with the colors representing the mean focusing depth, $\bar{z}_{f}$. The $\mathrm{x}$ and y axis correspond to distance from the ridge axis $(\mathrm{km})$ and depth $(\mathrm{km})$.

Table 3: Melt focusing analysis

\begin{tabular}{c|c|ccc|cccc|c} 
Symbol & Formula/Definition & \multicolumn{5}{|c|}{ Values } & Units \\
\hline$K_{0}$ & intrinsic permeability & \multicolumn{3}{|c|}{$4 \times 10^{-9}$} & \multicolumn{3}{|c}{$4 \times 10^{-7}$} & $\mathrm{~m}^{2}$ \\
\hline$U_{0}$ & half-spreading rate & 2 & 4 & 8 & 2 & 4 & 8 & $\mathrm{~cm} / \mathrm{yr}$ \\
\hline$z_{\text {focus }}$ & Equation 35 & 22.8 & 24.1 & 24.9 & 21.3 & 24.1 & 26.6 & $\mathrm{~km}$ \\
$L_{\text {melting }}$ & Equation 36 & 13.5 & 14.0 & 12.7 & 29.0 & 28.9 & 29.8 & $\mathrm{~km}$ \\
$Q_{\text {focus }}$ & Equation 37 & 0.33 & 0.26 & 0.33 & 0.43 & 0.49 & 0.49 & -
\end{tabular}

\subsection{Melt transport analysis}

To quantify the relative contributions of the different focusing mechanisms in the models (Figure 3), we analyze the non-dimensional melt flux profiles across an arc of $5 \mathrm{~km}$ radius centered at the ridge axis and the instantaneous flow lines emanating from it (Figure 8). The melt flux, $\mathbf{q}=\phi \mathbf{v}_{\mathbf{f}}$, is obtained using Equation 4 and $\hat{\mathbf{r}}$ is the unit normal to the arc. Together, the nondimensional melt flux normal to the arc, $\mathbf{q} \cdot \hat{\mathbf{r}}$ (red lines in all the i) panels in Figure 8), suggests two distinct sources of melt supply to the ridge axis: melt 
flux from the central region and melt flux from the decompaction layers on the sides. However, melt flux is only a local measure and it is not obvious given only $\mathbf{q}$ whether the melt has been focused at depth or traveled in shallow decompaction channels. To better quantify these ideas, we also calculate the instantaneous flow lines (all the ii) panels in Figure 8) that arrive at the sampling arc of $5 \mathrm{~km}$ radius centered at the ridge axis and calculate several metrics for each flow line that allow us to distinguish between deep and shallow melt focusing. The first measure is the extraction width, $\left|x_{f}\right|$, which is the distance from the axis that a melt streamline originates from at the solidus $\sim 60 \mathrm{~km}$ (dashed black lines in all the i) panels in Figure 8). We also plot the $\mathrm{x}$-coordinate on the arc of $5 \mathrm{~km}$ radius, $\left|x_{0}\right|$. The second measure is the integrated mean depth of focusing, $\bar{z}_{f}$, which is the depth weighted by the magnitude of the horizontal flux:

$$
\bar{z}_{f}=\frac{\int_{\text {flowline }} z q_{x} d \tau}{\int_{\text {flowline }} q_{x} d \tau}
$$

where $z$ and $q_{x}$ are the depth and the horizontal component of the melt flux at any point of the flow line and $\tau$ is the time travelled along the flow line, which goes to the dry solidus at about $60 \mathrm{~km}$ depths. The instantaneous flow lines are colored by $\bar{z}_{f}$ (all the ii) panels in Figure 8). Shallow horizontal transport in decompaction channels should lead to smaller $\bar{z}_{f}$ etc.

We compare $\mathbf{q},\left|x_{f}\right|,\left|x_{0}\right|$ and $\bar{z}_{f}$ for all the model runs (Figure 3 ) in the i) panels in Figure 8. Melt flux coming from either the central region and decompaction channels is clearly identifiable in all measures. In general, for these model runs, $\mathbf{q}$ is bi-modal with a central peak coming from deeper within the melting region and two side peaks coming from the shallower decompaction channels. Melt that ends up in the central region comes from 
a shorter horizontal distance from the ridge axis and has deeper mean melt focusing depth. On the other hand, melt that ends up in the decompaction channels come from further off the ridge axis and has shallower mean focusing depth. The peaks in flux at the sides become shallower with increasing spreading rate as expected with plate cooling.

In all these model cases, there is very little melt flux between the two peaks and a simple discriminate is to use the two minima, $\alpha_{-}$and $\alpha_{+}$, in melt flux on the arc (black triangles in all the i) panels in Figure 8). The "central melts" come from between these two minima while the "channel melts" come from beyond either minima. Given this separation, we can further quantify the width of melting pressure focusing in the central region:

$$
L_{\text {melting }}=\frac{\left|x_{f_{\alpha_{-}}}\right|+\left|x_{f_{\alpha_{+}}}\right|}{2}
$$

which is the length scale over which melting pressure focusing operates in these models. To quantify the melt flux coming from the decompaction channels or the central region, we define the melt flux quotient, $Q_{\text {focus }}$ :

$$
Q_{\text {focus }}=\frac{\int_{\alpha_{-}}^{\alpha_{+}} \mathbf{q} \cdot \hat{\mathbf{r}} d \alpha}{\int_{\alpha} \mathbf{q} \cdot \hat{\mathbf{r}} d \alpha}
$$

which tells us the proportions of total melt flux coming from the central region. Since this quotient does not discriminate how much this melt have been focused, we also define the mean focusing depth, $z_{\text {focus }}$, to be given by average of the two $\bar{z}_{f}$ at the two minima of normal melt flux, $\alpha_{-}$and $\alpha_{+}$ (black triangles in all the i) panels in Figure 8).

Table 3 shows how these different measures for melt focusing change with intrinsic permeability and spreading rate. Melting pressure focusing acts over the length scale, $L_{\text {melting, }}$, that is roughly twice the width at about $30 \mathrm{~km}$ for 
the model runs with larger intrinsic permeability while remaining unchanged with spreading rates (Table 3). The proportions of total melt flux coming from the central region, $Q_{\text {focus }}$, increases with intrinsic permeability and does not change significantly with spreading rates (Table 3). The mean focusing depth, $z_{\text {focus }}$, does not change significantly with intrinsic permeability or spreading rate (Table 3 ).

A corresponding figure for the rheological models shown in Figure S1 is given in the supplementary material. In general, there is a transition from decompaction layer focusing to melting pressure focusing in the isoviscous model cases consistent with Figure 7. There is stronger melting pressure focusing when dislocation creep is excluded (panel d) in Figure S1).

\subsection{Comparison with geophysical observations with implications for the litho-} sphere asthenosphere boundary (LAB)

The porosities, melt velocities and melt transport patterns from these models can be compared with geophysical observations from both seismic and electromagnetic surveys at various mid-ocean ridges. The models predict two high porosity regions, one on the ridge axis at depths (between 10 to $40 \mathrm{~km}$ depths) and another in the decompaction layers, following the LAB roughly. Geophysical estimates of porosity beneath the fast spreading East Pacific Rise (EPR) range from $<1 \%$ to $>10 \%$, although these estimates are sensitive to assumptions about how porosity affects observable seismic and electromagnetic properties (Forsyth et al., 1998b,a; Baba et al., 2006; Key et al., 2013). For faster spreading rate, the models predict up to $20 \%$ porosity for models with lower permeability and an order of magnitude lower for models with higher permeability. A model with lower permeability generally 
leads to slower melt velocities and hence larger amount of melt retention.

Both magnetotelluric (MT) imaging of the EPR and seismic attenuation studies at Juan de Fuca ridge suggest that melt may be focused deeper even before reaching the theorized decompaction layers (Key et al., 2013; Eilon and Abers, 2017; Ruan et al., 2018). The MT inversions show that the shallow upper mantle is resistive and devoid of melt (Key et al., 2013) where the decompaction layers are predicted to be in existing models while the seismic attenuating regions are much deeper than the expected decompaction layers as well (Eilon and Abers, 2017; Ruan et al., 2018). The melt rich decompaction layers essentially delineates the LAB in the models, which would otherwise by be defined by both the temperature dependent rheological or freezing boundaries. The prominent decompaction layers in the models with smaller intrinsic permeability, $K_{0}=4 \times 10^{-9} \mathrm{~m}^{2}$ (Figure 3), make them perhaps less plausible since higher porosities are better detected by geophysical methods. It is also possible that there exist melt channels that transport melt rapidly along this decompaction layer that are too small and narrow to be detected by geophysical observations since the porosity shown in our models are volumetric averages of each mesh element $(\sim 1 \mathrm{~km}$ along decompaction layers). Although the MT method is sensitive to conductors such as melt, the ocean is highly conductive and attenuates the high frequency natural source field such that seafloor MT data may not be able to resolve narrow and shallow melt channels. However, if the interpretations of the MT surveys are accurate, one possibility to reconcile this discrepancy would be that the melting pressure focusing is even stronger in reality compared to our models, since it is the only mechanism in our models thus far that focuses 
melt in the deeper melting region before it reaches the thermal boundary layer.

To strengthen the melting rate focusing mechanism, bulk viscosity has to be larger since melting is mainly controlled by solid mantle upwelling, which is better constrained. In this study, bulk viscosity depends on both shear viscosity and permeability, which sets the mean porosity retained (Equations 18 and 24). Similar models with grain size evolution considerations could reconcile this discrepancy (Turner et al., 2017) as there is a steeper freezing front in those models. Another way to reconcile this could be that the lithosphere is indeed thicker than previously thought. Turner et al. (2017) has shown that using different choices for rheological flow laws could lead to a thicker lithosphere.

\subsection{Model caveats}

Our models are one-way coupled so that the solid velocity and dynamic pressure in the solid system are not affected by the melt flow system. This problem is less computationally expensive since we avoid the need to solve the full Stokes equation at every time-step, while still capturing the main dynamics of the system. However, one-way coupling leads to an inconsistency in mass conservation. The solid velocity is not adjusted to move up faster as melt is produced and extracted/segregated. This is a minor contribution and may allow slightly more melt to be produced than predicted in Figure 4a.

Our numerical models are passively driven by a Dirichlet condition that sets the solid velocity at the top boundary to be plate spreading rate. Geophysical observations of asymmetric upwelling could suggest buoyancy driven flow beneath some mid-ocean ridges (Hammond and Toomey, 2003; Dunn 
and Forsyth, 2003; Baba et al., 2006). However, a combined geochemical and dynamical study suggests that passively driven upwelling seems more consistent with indirect geochemical observations (Spiegelman and Reynolds, 1999). Furthermore, symmetry in a highly conductive region beneath the East Pacific Rise is interpreted to be evidence for passively driven flow (Key et al., 2013).

\section{Conclusion}

We describe and present an openly available suite of two-phase flow models applied to mid-ocean ridge setting, varying half-spreading rates and intrinsic permeability to understand melt focusing. Three distinct melt focusing mechanisms are recognized in the models: 1) Melting pressure focusing, 2) Decompaction layers and 3) Ridge suction, of which the first two are dominant. Our models suggest that even with similar melting patterns, the amount of melt and melt transport patterns can be significantly different due to changes in intrinsic permeability, $K_{0}$, regardless of spreading rates: 1 ) increasing intrinsic permeability increases melt velocity, therefore decreasing porosity or melt fractions due to efficient melt transport, 2) this reduction in porosity then leads to an increase in bulk viscosity since the bulk viscosity model used here assumes $\zeta \approx \eta / \phi$. This increases the magnitude of the compaction pressure at quasi-steady state (Equation 34). In particular, the increase in compaction pressure and its gradient in the axial melting region pulls melt from a wider region towards the axis, which is the basis for melting pressure focusing. These melt focusing mechanisms are a natural consequence of the two phase flow formulation with viscous deformation and 
their manifestation depends largely on the rheological model choices made. Melting-pressure focusing is a consequence of the compaction pressure field mapping to the melting rate field modulated by the bulk viscosity in quasisteady state (Equation 34). The geometry of the melting rate field will always be roughly triangular in a ridge setting. However, the magnitude of the compaction pressure depends on the rheological model used for bulk viscosity and therefore shear viscosity in our models. Stronger overall shear viscosity in the melting region leads to larger melting pressure focusing effect. The length scale associated with melting pressure focusing is about two times wider for larger intrinsic permeability and the consequent increase in bulk viscosity. The dominance and strength of these melt focusing mechanisms affect the locality of melt rich regions and also melt transport, thus can affect the interpreted position of the LAB. To reconcile the models with geophysical observations with regards to the LAB, stronger melting pressure focusing might be needed to focus melt before it reaches the lithosphere.

\section{Acknowledgements}

We thank Tobias Keller and two anonymous reviewers for their constructive comments and suggestions. C.W.'s and M.S.'s work on TerraFERMA has been supported by NSF grants OCE-0841079, EAR-1141976, and OCE1358091, as well as by the Deep Carbon Observatory. S.S. and D.S were supported by National Science Foundation Grant No. EAR-1255040, and the Extreme Science and Engineering Discovery Environment (XSEDE), which is supported by National Science Foundation grant number ACI-1053575. We thank Donna Blackman, Steve Constable, Kerry Key, Samer Naif and James 
777

Day for useful discussions.

\section{References}

Asimow, P.D., Stolper, E., 1999. Steady-state mantle-melt interactions in one dimension: I. equilibrium transport and melt focusing. Journal of Petrology 40, 475-494.

Baba, K., Chave, A.D., Evans, R.L., Hirth, G., Mackie, R.L., 2006. Mantle dynamics beneath the east pacific rise at $17 \mathrm{~s}$ : Insights from the mantle electromagnetic and tomography (melt) experiment. Journal of Geophysical Research: Solid Earth 111.

Balay, S., Abhyankar, S., Adams, M.F., Brown, J., Brune, P., Buschelman, K., Dalcin, L., Eijkhout, V., Gropp, W.D., Kaushik, D., Knepley, M.G., May, D.A., McInnes, L.C., Rupp, K., Sanan, P., Smith, B.F., Zampini, S., Zhang, H., Zhang, H., 2017. PETSc Users Manual. Technical Report ANL-95/11 - Revision 3.8. Argonne National Laboratory. URL: http://www.mcs.anl.gov/petsc.

Bown, J.W., White, R.S., 1994. Variation with spreading rate of oceanic crustal thickness and geochemistry. Earth and Planetary Science Letters 121, 435-449.

Buck, W.R., Su, W., 1989. Focused mantle upwelling below mid?ocean ridges due to feedback between viscosity and melting. Geophysical Research Letters $16,641-644$. 
Carbotte, S.M., Smith, D.K., Cannat, M., Klein, E.M., 2016. Tectonic and magmatic segmentation of the global ocean ridge system: A synthesis of observations. Geological Society, London, Special Publications 420, 249295.

Dunn, R.A., Forsyth, D.W., 2003. Imaging the transition between the region of mantle melt generation and the crustal magma chamber beneath the southern east pacific rise with short-period love waves. Journal of Geophysical Research: Solid Earth 108.

Eilon, Z.C., Abers, G.A., 2017. High seismic attenuation at a mid-ocean ridge reveals the distribution of deep melt. Science advances 3, e1602829.

Fischer, K.M., Ford, H.A., Abt, D.L., Rychert, C.A., 2010. The lithosphereasthenosphere boundary. Annual Review of Earth and Planetary Sciences $38,551-575$.

Forsyth, D.W., Scheirer, D.S., Webb, S.C., Dorman, L.M., 1998a. Imaging the deep seismic structure beneath a mid-ocean ridge: The MELT experiment. Science , 1-5.

Forsyth, D.W., Webb, S.C., Dorman, L., Shen, Y., 1998b. Phase velocities of Rayleigh waves in the MELT experiment on the East Pacific Rise. Science , $1-5$.

Fowler, A., 1985. A mathematical model of magma transport in the asthenosphere. Geophysical \& Astrophysical Fluid Dynamics 33, 63-96. 
Geuzaine, C., Remacle, J.F., 2009. Gmsh: A 3-d finite element mesh generator with built-in pre-and post-processing facilities. International journal for numerical methods in engineering 79, 1309-1331.

Ham, D., Farrell, P., Gorman, G., Maddison, J., Wilson, C., Kramer, S., Shipton, J., Collins, G., Cotter, C., Piggott, M., 2009. Spud 1.0: generalising and automating the user interfaces of scientific computer models. Geoscientific Model Development 2, 33.

Hammond, W.C., Toomey, D.R., 2003. Seismic velocity anisotropy and heterogeneity beneath the mantle electromagnetic and tomography experiment (melt) region of the east pacific rise from analysis of $\mathrm{p}$ and $\mathrm{s}$ body waves. Journal of Geophysical Research: Solid Earth 108.

Harding, J.L., Van Avendonk, H.J., Hayman, N.W., Grevemeyer, I., Peirce, C., Dannowski, A., 2017. Magmatic-tectonic conditions for hydrothermal venting on an ultraslow-spread oceanic core complex. Geology 45, 839-842.

Hebert, L.B., Montési, L.G., 2010. Generation of permeability barriers during melt extraction at mid-ocean ridges. Geochemistry, Geophysics, Geosystems 11 .

Hewitt, I., Fowler, A., 2008. Partial melting in an upwelling mantle column, in: Proceedings of the Royal Society of London A: Mathematical, Physical and Engineering Sciences, The Royal Society. pp. 2467-2491.

Hirschmann, M.M., 2000. Mantle solidus: Experimental constraints and the effects of peridotite composition. Geochemistry Geophysics Geosystems 1, $\mathrm{n} / \mathrm{a}-\mathrm{n} / \mathrm{a}$. 
Hirth, G., Kohlstedt, D., 2003. Rheology of the upper mantle and the mantle wedge: A view from the experimentalists. Inside the subduction Factory , 83-105.

Holtzman, B., Groebner, N., Zimmerman, M., Ginsberg, S., Kohlstedt, D., 2003a. Stress-driven melt segregation in partially molten rocks. Geochemistry, Geophysics, Geosystems 4.

Holtzman, B., Kohlstedt, D., Zimmerman, M., Heidelbach, F., Hiraga, T., Hustoft, J., 2003b. Melt segregation and strain partitioning: implications for seismic anisotropy and mantle flow. Science 301, 1227-1230.

Karato, S.i., Wu, P., 1993. Rheology of the upper mantle: a synthesis. Science 260, 771-778.

Katz, R.F., 2008. Magma Dynamics with the Enthalpy Method: Benchmark Solutions and Magmatic Focusing at Mid-ocean Ridges. Journal of Petrology 49, 2099-2121.

Katz, R.F., Knepley, M.G., Smith, B., Spiegelman, M., Coon, E.T., 2007. Numerical simulation of geodynamic processes with the portable extensible toolkit for scientific computation. Physics of the Earth and Planetary Interiors $163,52-68$.

Katz, R.F., Spiegelman, M., Holtzman, B., 2006. The dynamics of melt and shear localization in partially molten aggregates. Nature 442, 676-679.

Katz, R.F., Spiegelman, M., Langmuir, C.H., 2003. A new parameterization of hydrous mantle melting. Geochemistry, Geophysics, Geosystems 4. 
Kawakatsu, H., Utada, H., 2017. Seismic and electrical signatures of the lithosphere-asthenosphere system of the normal oceanic mantle. Annual Review of Earth and Planetary Sciences 45.

Keller, T., Katz, R.F., 2016. The Role of Volatiles in Reactive Melt Transport in the Asthenosphere. Journal of Petrology 57, 1073-1108.

Keller, T., Katz, R.F., Hirschmann, M.M., 2017. Earth and Planetary Science Letters. Earth and Planetary Science Letters 464, 55-68.

Key, K., Constable, S., Liu, L., Pommier, A., 2013. Electrical image of passive mantle upwelling beneath the northern East Pacific Rise. Nature 495, 499-502.

Kohlstedt, D.L., Holtzman, B.K., 2009. Shearing melt out of the earth: An experimentalist's perspective on the influence of deformation on melt extraction. Annual Review of Earth and Planetary Sciences 37, 561-593.

Logg, A., Mardal, K.A., Wells, G., 2012. Automated solution of differential equations by the finite element method: The FEniCS book. volume 84 . Springer Science \& Business Media.

Logg, A., Ølgaard, K., Rognes, M., Wells, G., Jansson, J., Kirby, R., Knepley, M., Lindbo, D., Skavhaug, O., 2011. The fenics project. GNU Free Documentation License 1.

Macdonald, K.C., 1982. Mid-ocean ridges: Fine scale tectonic, volcanic and hydrothermal processes within the plate boundary zone. Annual Review of Earth and Planetary Sciences 10, 155-190. 
McKenzie, D., 1984. The Generation and Compaction of Partially Molten Rock. Journal of Petrology , 1-53.

Miller, K.J., Zhu, W.l., Montési, L.G.J., Gaetani, G.A., 2014. Experimental quantification of permeability of partially molten mantle rock. Earth and Planetary Science Letters 388, 273-282.

Moresi, L., Quenette, S., Lemiale, V., Meriaux, C., Appelbe, B., Mühlhaus, H.B., 2007. Computational approaches to studying non-linear dynamics of the crust and mantle. Physics of the Earth and Planetary Interiors 163, 69-82.

Morgan, J.P., 1987. Melt migration beneath mid?ocean spreading centers. Geophysical Research Letters 14, 1238-1241.

Ribe, N.M., 1985. The deformation and compaction of partial molten zones. Geophysical Journal International 83, 487-501.

Ribe, N.M., 1987. Theory of melt segregation:a review. Journal of volcanology and geothermal research 33, 241-253.

Ruan, Y., Forsyth, D.W., Bell, S.W., 2018. Shear attenuation beneath the juan de fuca plate: Implications for mantle flow and dehydration. Earth and Planetary Science Letters 496, 189-197.

Rudge, J.F., 2018a. Textural equilibrium melt geometries around tetrakaidecahedral grains. Proceedings of the Royal Society A: Mathematical, Physical and Engineering Sciences 474, 20170639. 
Rudge, J.F., 2018b. The viscosities of partially molten materials undergoing diffusion creep. Journal of Geophysical Research: Solid Earth 123, 10-534.

Scott, D.R., Stevenson, D.J., 1984. Magma solitons. Geophysical Research Letters 11, 1161-1164.

Scott, D.R., Stevenson, D.J., 1986. Magma ascent by porous flow. Journal of Geophysical Research: Solid Earth 91, 9283-9296.

Simpson, G., Spiegelman, M., Weinstein, M.I., 2010a. A multiscale model of partial melts: 1. effective equations. Journal of Geophysical Research: Solid Earth 115.

Simpson, G., Spiegelman, M., Weinstein, M.I., 2010b. A multiscale model of partial melts: 2. numerical results. Journal of Geophysical Research: Solid Earth 115.

Sparks, D.W., Parmentier, E.M., 1991. Melt extraction from the mantle beneath spreading centers. Earth and Planetary Science Letters 105, 368377.

Spiegelman, M., 1993a. Flow in deformable porous media. Part 1: Simple analysis. Journal of Fluid Mechanics 247, 17-38.

Spiegelman, M., 1993b. Flow in deformable porous media. part 2: numerical analysis-the relationship between shock waves and solitary waves. Journal of Fluid Mechanics 247, 39-63.

Spiegelman, M., 1993c. Physics of Melt Extraction: Theory, Implications and 
Applications. Philosophical Transactions of the Royal Society A: Mathematical, Physical and Engineering Sciences 342, 23-41.

Spiegelman, M., 1996. Geochemical consequences of melt transport in 2-D: The sensitivity of trace elements to mantle dynamics. Earth and Planetary Science Letters 139, 115-132.

Spiegelman, M., Elliott, T., 1993. Consequences of melt transport for uranium series disequilibrium in young lavas. Earth and Planetary Science Letters .

Spiegelman, M., May, D.A., Wilson, C.R., 2016. On the solvability of incompressible Stokes with viscoplastic rheologies in geodynamics. Geochemistry Geophysics Geosystems 17, 2213-2238.

Spiegelman, M., McKenzie, D., 1987. Simple 2-D models for melt extraction at mid-ocean ridges and island arcs. Earth and Planetary Science Letters .

Spiegelman, M., Reynolds, J.R., 1999. Combined dynamic and geochemical evidence for convergent melt flow beneath the east pacific rise. Nature 402, $282-285$.

Tosi, N., Stein, C., Noack, L., Hüttig, C., Maierova, P., Samuel, H., Davies, D.R., Wilson, C.R., Kramer, S.C., Thieulot, C., et al., 2015. A community benchmark for viscoplastic thermal convection in a 2-d square box. Geochemistry, Geophysics, Geosystems 16, 2175-2196. 
Turner, A.J., Katz, R.F., Behn, M.D., Keller, T., 2017. Magmatic Focusing to Mid-Ocean Ridges: The Role of Grain-Size Variability and NonNewtonian Viscosity. Geochemistry Geophysics Geosystems 23, 15.

Vera, E., Mutter, J., Buhl, P., Orcutt, J., Harding, A., Kappus, M., Detrick, R., Brocher, T., 1990. The structure of 0-to 0.2-my-old oceanic crust at $9 \mathrm{n}$ on the east pacific rise from expanded spread profiles. Journal of Geophysical Research: Solid Earth 95, 15529-15556.

Wark, D.A., Watson, E.B., 1998. Grain-scale permeabilities of texturally equilibrated, monomineralic rocks. Earth and Planetary Science Letters 164, 591-605.

White, R.S., McKenzie, D., O'Nions, R.K., 1992. Oceanic crustal thickness from seismic measurements and rare earth element inversions. Journal of Geophysical Research: Solid Earth 97, 19683-19715.

White, R.S., Minshull, T.A., Bickle, M.J., Robinson, C.J., 2001. Melt generation at very slow-spreading oceanic ridges: Constraints from geochemical and geophysical data. Journal of Petrology 42, 1171-1196.

Wilson, C.R., Spiegelman, M., van Keken, P.E., 2017. TerraFERMA: The Transparent Finite Element Rapid Model Assembler for multiphysics problems in Earth sciences. Geochemistry Geophysics Geosystems 18, 769-810.

Wilson, C.R., Spiegelman, M., van Keken, P.E., Hacker, B.R., 2014. Fluid flow in subduction zones: The role of solid rheology and compaction pressure. Earth and Planetary Science Letters 401, 261-274. 
971 Zhu, W., David, C., Wong, T., 1995. Network modeling of permeability 972 evolution during cementation and hot isostatic pressing. Journal of Geo973 physical Research . 